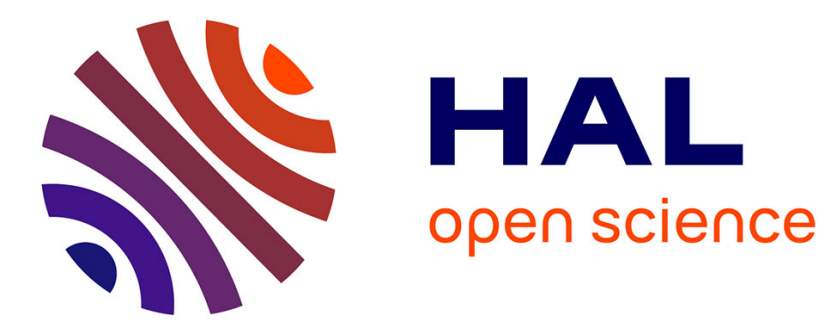

\title{
Des pratiques et des goûts culturels bourgeois pendant la Révolution \\ Philippe Bourdin
}

\section{To cite this version:}

Philippe Bourdin. Des pratiques et des goûts culturels bourgeois pendant la Révolution. Jean-Pierre Jessenne. Révolution française et changement social: vers un ordre bourgeois, Presses universitaires de Rennes, pp.317-342, 2007. halshs-01762939

\section{HAL Id: halshs-01762939 \\ https://shs.hal.science/halshs-01762939}

Submitted on 10 Apr 2018

HAL is a multi-disciplinary open access archive for the deposit and dissemination of scientific research documents, whether they are published or not. The documents may come from teaching and research institutions in France or abroad, or from public or private research centers.
L'archive ouverte pluridisciplinaire $\mathbf{H A L}$, est destinée au dépôt et à la diffusion de documents scientifiques de niveau recherche, publiés ou non, émanant des établissements d'enseignement et de recherche français ou étrangers, des laboratoires publics ou privés. 


\section{Des pratiques et des goûts culturels bourgeois pendant la Révolution}

Source : Jean-Pierre JESSENNE (dir.), Actes du colloque de Lille (12, 13, 14 janvier 2006), Révolution française et changement social : vers un ordre bourgeois, Lille, Presses universitaires du Septentrion, 2007, p. 317-342.

Résumé : Il ne s'agit pas, dans l'espace limité d'un article, de passer en revue les différents champs dans lesquels les diverses strates de la bourgeoisie française ont pu, durant la décennie 1789-1799, exercer leurs appétits ou leurs appétences. Mais, partant d'un aperçu sur le goût alimentaire, d'inviter à un voyage forcément impressionniste qui déclinera les goûts vestimentaires et partie des goûts intellectuels et artistiques en essayant de montrer les critères de la distinction "bourgeoise", leurs attendus politiques et sociaux, dans le prolongement d'une identité ou d'identités affirmées durant le siècle.

Mots clés : bourgeoisie, goût, alimentation, loisirs, peinture, mode, théâtre, amateurs

Les deux termes principaux du sujet (la bourgeoisie, le goût) paraissent autant de pièges pour le chercheur tant leur définition est tout sauf évidente. De manière significative, dans l'ensemble de portraits peints dans l'atelier de Michel Vovelle, qui révèlent L'homme des Lumières, le noble et le prêtre ont droit de cité, non le bourgeois, partagé sans doute entre l'homme d'affaires, l'homme de lettres ou de sciences, le fonctionnaire ${ }^{1}$. Dans son essai de définition de la nouvelle classe politique issue de la Révolution, un aréopage majoritairement quadragénaire et urbain aux contours sociaux divers et fluctuants selon les Constitutions et le système électoral, au sein duquel elle compte «bourgeois Jacobins» et «petits bourgeois sans-culottes», Lynn Hunt souligne le poids des réseaux (professionnels, familiaux, de voisinage ou de club) et de la culture ${ }^{2}$. Mais, reprenant les éléments de définition des classes par Marx, elle pointe tout autant la difficulté à discerner la conscience politique de la bourgeoisie révolutionnaire à partir d'un certain nombre de valeurs et de positions politiques diffusées par nombre d'intermédiaires (rationalisme, exaltation de la nation, lutte contre la féodalité, l'absolutisme, l'aristocratie, par exemple) : ses adversaires ont pu un temps ou en partie les partager; elles s'avèrent insuffisantes pour préserver quelque unité que ce soit durant toute la décennie. Le croisement des biographies accentue cette complexité. Encore est-il question ici de culture politique qui, si elle influe sur les goûts privés et collectifs, ne saurait les délimiter.

Du goût, l'Encyclopédie donne idée de la diversité des acceptions :

«Le goût est de toutes les sensations : on a du goût pour la Musique \& pour la Peinture, comme pour les ragoûts, quand l'organe de ces sensations savoure, pour ainsi dire, ces objets. [...] Comme le mauvais goût au physique consiste à n'être flatté que par des assaisonnemens trop piquans \& trop recherchés, aussi le mauvais goût dans les Arts est de ne se plaire qu'aux ornemens étudiés, \& de ne pas sentir la belle nature. [...] Le goût se forme insensiblement dans une nation qui n'en avoit pas, parce qu'on y prend peu-à-peu l'esprit des bons artistes : on s'accoûtume à voir des tableaux avec les yeux de Lebrun, du Poussin, de le Sueur ; on entend la déclamation notée des scenes de Quinaut avec l'oreille de Lulli ; \& les airs, les symphonies, avec celle de Rameau. On lit les livres avec l'esprit des bons auteurs ».

Sensation et éducation sont donc associées dans cet apprentissage du « bon goût » qui va faire les véritables «amateurs », terme tout autant problématique. Antoine Furetière parle de « celui qui aime quelque chose », offrant ensuite un territoire social et intellectuel des plus larges à cette appétence, quoique le domaine artistique ait une place privilégiée : "Il est amateur de l'étude, des curiosités, des tableaux, des coquilles, amateur de la Musique, des beaux-arts. Le peuple est amateur de nouveautez ». Paul-Émile Littré synthétisera les définitions contradictoires et les a priori

\footnotetext{
${ }^{1}$ Michel VovelLe, L'homme des Lumières, Paris, Seuil, 1996.

${ }^{2}$ Lynn Hunt, Politics, Culture and Class in the French Revolution, Berkeley, University of California Press, $2004\left(20^{\mathrm{e}}\right.$ édition), p. 150 : «The new political class was defined as much by its cultural positions and relationships as by its membership in occupationally defined social groups ».
} 
à l'œuvre dès le XVIII ${ }^{\mathrm{e}}$ siècle, de l'épithète flatteuse à la plus calamiteuse — en 1738 à Paris, les statuts de la corporation des peintres et sculpteurs ne précisent-ils pas que la défense du monopole du dessin exclut les «bourgeois désireux de travailler de leurs mains lesdits Arts, mais pour leurs usages seulement, et non pour en faire vente ou commerce $»^{3}$. Littré ne fait plus seulement du goût une inclination mais dans la circonstance le lie à une pratique, qu'elle soit du domaine du loisir ou de la profession, de l'obsession amoureuse ou de la médiocrité nonchalante. La pratique connote donc l'«amateur»: «1 - Celui qui a un goût vif pour une chose. Un amateur de peinture, de musique [...]. 2 - Celui qui cultive les beaux-arts sans en faire profession. C'est un amateur distingué $[\ldots] .3$ - C'est un amateur, c'est un homme de talent médiocre. 4 - Homme s'occupant peu de son métier [...]. Être amateur indique toujours une préférence particulière et devenue, en quelque sorte, une étude: je suis amateur de roses signifie que je les recherche, que j'en fais collection ». Notons la dérive ainsi enregistrée dans les objets d'une curiosité.

Il ne s'agit pas, dans l'espace limité d'un article, de passer en revue les différents champs dans lesquels les diverses strates de la bourgeoisie française ont pu, durant la décennie 1789-1799, exercer leurs appétits ou leurs appétences. Mais, partant d'un aperçu sur le goût alimentaire, dont découlent, on l'aura compris, toutes les définitions, d'inviter à un voyage forcément impressionniste qui déclinera les goûts vestimentaires et partie des goûts intellectuels et artistiques - en faisant taire, à notre grand dam, la musique -, en essayant de montrer les critères de la distinction « bourgeoise », leurs attendus politiques et sociaux, dans le prolongement d'une identité ou d'identités affirmées durant le siècle.

\section{Les plaisirs des papilles}

L'importance du goût alimentaire se retrouve dans son emploi métaphorique, résumant l'appétence pour l'ensemble des arts, telle que la résume Voltaire en 1764, dans son Dictionnaire philosophique: «Le goût, ce sens, ce don de discerner nos alimens, a produit dans toutes les langues connues la métaphore qui exprime, par le mot goût, le sentiment des beautés et des défauts dans tous les arts : c'est un discernement prompt, comme celui de la langue et du palais, et qui prévient comme lui la réflexion ; il est comme lui sensible et voluptueux à l'égard du bon ; il rejette, comme lui, le mauvais avec soulèvement ». L'évolution lexicale montre comment l'appréhension $\mathrm{du}$ goût (souvent confondu avec la saveur), davantage valorisé, s'enrichit aux XVII ${ }^{\mathrm{e}}$ et XVIII ${ }^{\mathrm{e}}$ siècles, usant d'un vocabulaire précisé et plus étendu qu'au Moyen-Âge : seize mots environ sont désormais employés, au lieu de six auparavant, dont bon, mauvais, délicat, délicieux, meilleur, excellent, fin, raffiné, fade, insipide, friand. Si la verdeur, le piquant, la pointe, reconnus dans les traités médiévaux, se perdent au profit du terme plus vague d' " amertume », si la salaison est moins finement perçue, la couleur, autrefois retenue pour son aspect décoratif, l'est désormais aussi pour ce qu'elle révèle de la nature du mets et de sa cuisson : blanc ou jaune d'œuf, blanc ou vert de poireau, beurre blanc ou roux, aloyau «saigneux et rouge », etc ${ }^{4}$. Des traités de cuisine sont venus les premiers essais de définition du «bon goût », chez L.S.R., dans L'Art de bien traiter (1674) ou Massaliot, dans son Cuisiner royal et bourgeois (1691), l'un et l'autre opposant civilité de la Cour et manières roturières, la première voulant que les recettes princières soient abandonnées sitôt imitées. Quoique les curiosités du financier Béchameil (1630-1703) prouvent que se développe un art gastronomique bourgeois, Grimod de la Reynière (1758-1838), d'une famille de fermiers généraux, continue de le mépriser, préférant ses dîners extravagants puis, sous l'Empire, ses jurys dégustateurs, qui réunissent entre autres Cambacérès, le marquis de Cussy ou le médecin gastronome Gastaldy, selon une sociabilité de salon qui fait de nombreuses victimes parmi les fournisseurs jugés ; mais le maître de cérémonie a l'habitude de ces conflits qu'il entretient dans ses gazettes, l'Almanach des gourmands (1803) ou le Manuel des amphytrions (1808). Dans sa Physiologie du goût, Brillat-Savarin (1755-1826), entérinant les exclusions nées de la recherche de

\footnotetext{
${ }^{3}$ Nathalie Heinich

${ }^{4}$ Jean-Louis FLANDRIN, «La distinction par le goût », in Philippe ARIES et Georges DUBY (direction), Histoire de la vie privée, tome III, Paris, Seuil, 1986, p. 286.
} 
distinction, établit pour sa part une série d' "éprouvettes gastronomiques », menus destinés à emporter l'adhésion des convives en fonction de leur milieu social : «Ainsi, l'éprouvette destinée à un petit rentier de la rue Coquenard ne fonctionnerait déjà plus chez un second commis, et ne s'apercevrait même pas à un dîner d'élus chez un financier ou un ministre $»^{5}$. Comme la fourchette des revenus pris en compte s'établit entre $5000 \mathrm{~F}$ («médiocrité ») — ce qui donne droit au dindon, aux pigeons bardés, à la choucroute et aux œufs à la neige -, et 30000 et plus («richesse ») s'alignent alors les volailles truffées (dinde, cailles) ou non (ortolans), le foie gras et le pâté de foie, la carpe et le brochet, les asperges et « une pyramide de meringue à la vanille et à la rose ${ }^{6}-$, on imagine que peu de "petits rentiers " ont accès à ces jeux forts qui, de part et d'autre de la Révolution, continuent d'associer les oligarchies. Encore Brillat-Savarin se réjouit-il des nouveautés, dont il fait abusivement des généralités, apportées par le règne de Louis XVI et la décennie révolutionnaire : la multiplication des professions de bouche, augmentées de nouvelles spécialités (les «pâtissiers de petit four»), les progrès techniques de la conservation, de l'horticulture (rendant accessibles fruits tropicaux ou légumineuses nouvelles), la diversification des importations de vin et les adoptions de spécialités étrangères (« des mets [...] comme le karik et le beefsteak; des assaisonnements, comme le caviar et le soy; des boissons, comme le ponche, le négus, et autres »), la diffusion du café. Il conclut sur le développement de la convivialité de table, dont il ne fait plus une valeur exclusivement noble ou bourgeoise :

«Les savants les plus distingués n'ont point cru au-dessous d'eux de s'occuper de nos premiers besoins, et ont introduit des perfectionnements, depuis le simple pot-au-feu de l'ouvrier jusqu'à ces mets extractifs et transparents qui ne sont servis que dans l'or ou le cristal [...].

On a commencé à séparer la gourmandise de la voracité et de la goinfrerie : on l'a regardée comme un penchant qu'on pouvoit avouer, comme une qualité sociale, agréable à l'amphytrion, profitable au convive, utile à la science, et on a mis les gourmands à côté de tous les autres amateurs qui ont aussi un objet connu de prédilection.

Un esprit général de convivialité s'est répandu dans toutes les classes de la société ; les réunions se sont multipliées, et chacun, en régalant ses amis, s'est efforcé de leur offrir ce qu'il avait remarqué de meilleur dans les zones supérieures.

[...] On a créé les banquets politiques, qui ont constamment eu lieu depuis trente ans toutes les fois qu'il a été nécessaire d'exercer une influence actuelle sur un grand nombre de volontés ; repas qui exigent une grande chère, à laquelle on ne fait pas attention, et où le plaisir n'est compté que pour mémoire.

Enfin, les restaurateurs ont paru : institution tout à fait nouvelle qu'on n'a point assez méditée, et dont l'effet est tel, que tout homme qui est maître de trois ou quatre pistoles peut immédiatement, infailliblement et sans autre peine que celle de désirer, se procurer toutes les jouissances positives dont le goût est susceptible ${ }^{7}$.

De fait, avec la Révolution, de bons cuisiniers de la cour et de la noblesse perdent leurs maîtres, émigrés, guillotinés, désargentés, et choisissent de fonder leurs propres maisons (Méot ou Robert, autrefois au service de Condé, Bancelin, Henneveu, Véry), privilégiant à Paris le quartier du Palais-Royal, où ils vont accueillir nombre de députés provinciaux. Si l'on en compte une centaine avant 1789 , il y aura près de six cents restaurants dans la capitale sous l'Empire, et la mode s'est répandue en province au profit des commerçants, des artisans, des fonctionnaires et des rentiers ${ }^{8}$. La multiplication de ces maisons offre à leurs hôtes des cartes riches de plats savamment montés, de vins savamment choisis, qui bouleversent l'art de cuisiner et rend caduques les anciens livres de recettes: «après 1800, aucun texte d'avant 1789 ne sera repris par les éditeurs français, à l'exception de La Cuisinière bourgeoise » (publiée par Menon en 1746) qui, comme Le Parfait Cuisinier français, édité en l'an VII par les Libraires associés, tente de répondre aux attentes du plus grand nombre de lecteurs - distinguant notamment morceaux dignes et indignes, la « basse boucherie » étant abandonnée au « bas peuple ${ }^{9} \ldots$ La production révolutionnaire frappe par son souci de vulgarisation, que prouve le Manuel du Cuisinier amateur (1798) ou La Cuisinière républicaine, ouvrage dans lequel Mme Mérigot, digne émule de Parmentier ou des amateurs

\footnotetext{
${ }^{5}$ BRILlat-SAVARIN, Physiologie du goût, édition présentée par Jean-François Revel, Paris, Flammarion, 1982 , p. 164.

${ }^{6}$ Ibidem, p. 167.

${ }^{7}$ Brillat-SaVArin, Physiologie du goût, p. 275.

${ }^{8}$ Jean-Louis FLANDRIN et Massimo MONTANARI (direction), Histoire de l'alimentation, Paris, Fayard, 1996, p. 772.

${ }^{9}$ Ibidem, p. 655.
} 
européens du tubercule vantés par Engel dans le Supplément de l'Encyclopédie ${ }^{10}$, propose en l'an III différentes manières d'accommoder la pomme de terre, avec ce seul credo : "la simplicité et l'économie ${ }^{11}$. Un deuxième souci, d'ordre diététique, transparaît au moins dans les titres : $L a$ Cuisine de santé (1789), La Pâtisserie de santé (1790), de Le Cointe et Jourdan ; il est conduit par l'adaptation aux contraintes du temps dans Le Petit cuisinier économe (an IV), au contraire du Manuel de la friandise (an V). Car la petite bourgeoisie subit aussi les privations qu'imposent les crises agricoles et la situation économique de la République. Dans sa correspondance, Nicolas Ruault, libraire parisien admirateur et éditeur de Voltaire, se plaint de la hausse vertigineuse des prix en floréal an II, qui le conduit à partager avec le peuple de la capitale les distributions quotidiennes de «tout au plus deux onces de pain par tête avec une cuillerée de riz, et quel pain, grand Dieu ! Celui que l'on donnait aux chiens, l'an passé [...] était incomparablement meilleur »; sa santé s'en ressent: "J'ai toujours mal aux yeux et des aigreurs à l'estomac » ${ }^{12}$. Mais la production des manuels répond aussi à l'amour maintenu d'une frange lettrée et aisée de la population pour l'art culinaire. Louis-Sébastien Mercier, dans son Nouveau Paris, note les excès de ces amateurs, dont il ne s'exonère pas, jusque dans les prisons de la Terreur - les sources provinciales confirment le fait, qui connait cependant d'importantes variantes selon les dates et l'activité des comités de surveillance et des représentants en mission ${ }^{13}-$ :

«Les victimes, dans les prisons, sacrifiaient à l'estomac, et l'étroit guichet voyait passer les viandes les plus exquises pour des hommes qui touchaient à leurs derniers repas et ne l'ignoraient point.

Du fond d'un cachot, on faisait un traité avec un restaurateur, et les articles signés de part et d'autre avec des conditions particulières sur les primeurs.

On ne visitait point un prisonnier sans lui apporter pour consolation la bouteille de Bordeaux, les liqueurs des îles et le plus délicat des pâtés.

De son côté, le pâtissier, qui sait très bien que la bouche va toujours, faisait descendre ses cartes jusqu'au fond des prisons [...]. Rien n'égale la délicatesse de son art, dit l'annonce : les ramequins et les meringues, en attestant les progrès de son industrie, attesteront ceux de la friandise parisienne, libre ou prisonnière.

Sa boutique vitrée est devenue plus belle, mieux décorée : elle est aussi nette dans l'intérieur que celle du bijoutier. Les tartelettes et les brioches y sont rangées sous verre avec autant de symétrie que des curiosités d'histoire naturelle. Il a fait une étude savante de tous les goûts, de tous les tempéraments.

Il faut qu'au coup d'œil appétissant de ces pâtés d'ortolans et de ces tourtes aux rognons, l'étranger, le fournisseur, le nouveau millionnaire, le captif par ouï-dire, fouillent à l'escarcelle. Qui le croira ? Lorsque le sang coulait à grands flots, le pâtissier, plus audacieux dans ses conceptions que l'Arétin, se mit à pétrir la pâte en priapes, et à donner à des gâteaux la forme du sexe virginal. Tous les excès se touchent : jamais l'on ne vit plus de propension à la gourmandise que dans ces jours de calamité et d'horreur : j'en atteste les six prisons où j'ai été plongé $»^{14}$.

La table indéniablement demeure le reflet du statut social, voire d'une ascension qui libère du souci alimentaire quotidien. Officier dans la garde nationale de sa section, restée fidèle à la Convention le $1^{\text {er }}$ prairial an III, le maître vitrier Ménétra se contente pour sa journée sous les drapeaux d'une livre de pain et d'un bon cervelas, mais son journal est scandé par les haltes nécessaires à sa sustentation, déjeuner ou souper, aux banquets compagnonniques, à ses plaisirs de guinguettes et d'auberges, autour d'une tête de veau, d'une volaille, de petit salé, d'une matelote, d'une salade et d'un gigot, accompagnés des vins de qualités variées que lui a fait découvrir son tour de France et boire jusqu'à l'ivresse les toasts fraternels. Il aime faire valoir, au soir de la

\footnotetext{
${ }^{10}$ Lucette PEROL, «Aux Amateurs, l'Agriculture reconnaissante », in Jean-Louis JAM (dir.), Les divertissements utiles. Des amateurs au XVIIIe siècle, Clermont-Ferrand, Presses universitaires Blaise-Pascal, 2000, p. 73-86.

11 Béatrice FINK (présentation), Les liaisons savoureuses. Réflexions et pratiques culinaires au dix-huitième siècle, Publications de l'Université de Saint-Étienne, 1995, p. 173-175.

${ }^{12}$ Christiane Rimbaud et Anne Vassal (présentation), Nicolas Ruault. Gazette d'un Parisien sous la Révolution. Lettres à son frère (1783-1796), Paris, Perrin, 1976, p. 379-382. Lettre du 22 floréal an III (11 mai 1795).

${ }^{13}$ Cf. Antoine-François DELANDINE, Tableau des prisons de Lyon pour servir à l'histoire de la tyrannie de 1792 et 1793, Lyon, 1797 ; MONTGEY et POIRIER, Les Angoisses de la mort, ou Idées et horreurs des prisons d'Arras, an III ; Jean-Baptiste Brochon, Histoire de mon arrestation et des événements qui me sont arrivés depuis la Société des Jeunes Gens, in Anne de MATHAN, Mémoires de la Terreur : l'an II à Bordeaux, Presses universitaires de Bordeaux, 2002 , p. 162.

${ }^{14}$ Louis-Sébastien Mercier, Le Nouveau Paris, an VII. Présenté par Michel DelON, Paris, Robert Laffont, 1990, p. 456.
} 
Révolution, qu'il vit «très bien »: «nous ne nous sentions point de la disette, nous étions pourvus, nous tenions bonne table ${ }^{15}$. On l'imagine donc manger à sa faim sans forcément goûter aux condiments et aux plats recherchés. De même, quand Dominique Doncre (1743-1820) portraiture en 1791 Le juge Pierre Lecocq et sa famille ${ }^{16}$ dans leur salle à manger, il montre certes la satisfaction et l'aisance d'une famille dont le père poursuit une carrière sans histoire, à défaut d'engagement fervent, mais, s'il insiste sur la richesse des panneaux peints, il ne présente du dîner que la soupière servie par un domestique. Lorsque, dans les années 1760, Chardin peignait La Pourvoyeuse, il en disait sans doute plus long sur les réalités de la petite et la moyenne bourgeoisies que bien des enthousiasmes de Brillat-Savarin : la quantité de pain, de vin rouge, de gibier, la fontaine à eau suggèrent un rang social, mais le peu de vaisselle empilé sur le buffet ne peut laisser supposer un service compliqué. Son Lièvre avec chaudron de cuivre, un coing et deux marrons, plaide pareillement pour des aliments simples, peu apprêtés ; la composition frugale du Bocal d'olives, ses assortiments de fruits, Le dessert de gaufrettes ou La brioche invitent plus à la dégustation sage et éclairée de produits de saison ou de recettes communes qu'à l'opulence des buffets à la française. Quand il remplit la table d'office (Les apprêts du déjeuner), l'abondance ne signifie d'ailleurs pas reniement de ces joies simples et des recettes de famille: salade et rôti, pâté, fromage, fruits, confitures, café et sucre n'entrent pas dans les «éprouvettes gastronomiques »... C'est d'ailleurs autour de plats semblables que le citoyen Romeau, sous le Directoire, invite ses concitoyens à fêter, en lieu et place de la Saint-Martin ou du Carnaval, les grandes heures de la Révolution dans La Tête ou l'oreille de cochon - deux morceaux de l'animal ostracisés depuis Louis XIV des assiettes des grands - :

«Le 14 juillet? Nos ingénieux pâtissiers sauront nous faire des Bastilles de biscuits, que nous nous empresserons de détruire avec le même zèle que nous avons vu nos brûlans Parisiens détruire cette lourde masse.

Le 10 août ? Nous y verrons le gros et gras poulet d'Inde figurer majestueusement sur nos tables, et nous rappeler cet imbécile parjure qui s'était imaginé que ses satellites résisteraient au courage du peuple, et qui, bientôt déçu de ses espérances, se trouva le dindon de sa téméraire entreprise.

Enfin, le 21 janvier serait partout caractérisé par la tête ou l'oreille de cochon, que chaque père de famille ne manquerait pas de mettre sur sa table, en mémoire du jour heureux où celle du parjure Louis XVI tomba, et nous délivra de sa triste présence.

[...] Ne perdons jamais de vue que c'est dans les repas de famille que l'on voit la gaité succéder à l'épanchement des cœurs et s'y associer à l'amour de sa patrie et de ses devoirs.

Il est malheureux, sans doute, que la fortune ne permette pas à tous nos frères indistinctement de mettre sur leurs tables une pièce aussi recommandable, aussi expressive, aussi commémorative que la tête ou l'oreille de cochon. Mais qu'ils se consolent de cet accident ; la providence n'a pas voulu que le pauvre soit privé de tout plaisir. Elle lui a donné, pour dédommagement de ce qu'elle lui a refusé, l'imagination avec laquelle il supplée à tout ce qui lui manque, et sans laquelle il n'existe pas une seule jouissance sur la terre. Oui, chez ceux là, le morceau de boudin, la saucisse, la crépinette même pourront remplacer la tête ou l'oreille, et il suffira d'une partie quelconque de l'animal pour être censé avoir participé à la fête $»^{17}$.

Ces jeux citoyens avec la nourriture auraient sans doute contrarié le Conventionnel Gilbert Romme, attaché à une ascèse privée; elle le ramenait au quotidien populaire qu'il aspirait à partager pour mieux le changer ${ }^{18}$. Non qu'il ignorât la variété des tables dans le milieu de robins auvergnats dont il était issu, partageant sa vie entre Riom (Puy-de-Dôme), siège d'intendance et de sénéchaussée, et, l'été venu, les campagnes voisines de Gimeaux. On sait par sa nièce, que les

\footnotetext{
15 Daniel Roche (présentation), Journal de ma vie de Jacques-Louis Ménétra, compagnon vitrier au XVIIIe siècle, Paris, Montalba, 1982, p. 271 et 280.

${ }^{16}$ Huile sur toile, 0,98 x 0,82 cm, in Philippe BORDES et Alain CHEVALIER, Catalogue des peintures, sculptures et dessins, Musée de la Révolution, Vizille, 1996, p. 71.

${ }^{17} \mathrm{BnF}, \mathrm{Lb}^{42}$ 912, RomeAu, La Tête ou l'oreille de cochon, Paris, Pellier, s.d., p. 3-4.

${ }^{18}$ Cf. Jean-Pierre Gross, Égalitarisme jacobin et droits de l'homme (1793-1794), Paris, Arcantères, 2000 , p. 203 et sqtes. Représentant en mission en Dordogne, Romme y imposera la mise en commun des grains dans des greniers communaux, la panification dans un four commun ou la distribution de farines pour la panification privée. Officiers et soldats disposent de la même ration (une livre et demie de pain, une livre de viande, une pinte de vin). Les ouvriers des mines, charbonnages et fonderies, donc des industries de guerre, se voient gratifiés d'un salaire de subsistance en pain, agrémenté de pommes de terre, riz, saindoux, vin et cochonnailles. Tous, riches et pauvres, mangent durant la période de soudure pendant laquelle brioches et pâtisseries sont interdites, le «pain de l'égalité », dont le député a fixé la teneur en son...
} 
agapes familiales n'ignoraient pas, en 1788, alors que le précepteur Romme avait amené en Auvergne son élève, le jeune comte russe Pavel Stroganov, les cuisses de grenouilles préparées par les traiteurs riomois, les produits de la chasse, de la pêche et de la cueillette, le veau et le mouton, les volailles de la basse-cour, le vin de Bourgogne, le champagne, les liqueurs anisées, le café, les fruits de toutes sortes, les friandises (la pâte d'abricot, notamment) ; que l'on partageait volontiers la soupe de pain blanc des mariages paysans du cru, comme les grands et cérémonieux services de la noblesse locale (ananas confit et curaçao viennent alors améliorer l'ordinaire) ${ }^{19}$. Inspiré par le modèle spartiate, Gilbert imposait pourtant déjà un strict régime à Pavel, le morigénant s'il acceptait des côtelettes en sauce : «la dernière vaisseleuse en saurait assez pour ce qu'il lui est permis de manger : du rotis, des légumes cuits à l'eau, des œufs frais, du laits et des fruits. Jamais de vin, encore moins de liqueur et pas de café $»^{20}$. En 1791, entré en Révolution comme d'autres entrent en religion, Romme donne lui-même l'exemple : il troque ses couverts d'argent, donnés à la patrie, contre d'autres en buis, mange des pommes de terre et partage son pain avec les pauvres ; au grand étonnement de ses proches, parfois admiratifs devant ce dévouement christique, «il s'impose toute espèce de privation $»^{21}$.

C'est là aller plus loin que bien des banquets républicains, concluant, quand le budget le permet, les cérémonies officielles ou, sous le Directoire, réunissant les électeurs - pratique du reste imitée en l'an V par l'Institut philanthropique. Que le repas puisse servir les négociations ou la propagande politiques est notoire, depuis le sommet du pouvoir, des antichambres de l'Assemblée aux comités de rédaction de certains journaux, tel Les Actes des Apôtres. Barras, qui est en train de promouvoir Bonaparte, se piquera de tenir table ouverte dans le Paris de l'an IV, échangeant ces bons services avec sa voisine, $\mathrm{M}^{\text {elle }}$ Montansier, désormais vieille gloire du théâtre français : "Forcé de dire quelquefois, comme mon cousin Lauraguais : "Ma table est ouverte, mais toujours couverte ", j'étais cependant assez heureux pour recevoir beaucoup de patriotes qui, ayant eu des rapports avec moi dans différentes positions révolutionnaires, pouvaient être assez peu gênés pour me révéler avec franchise qu'ils avaient besoin d'un dîner ${ }^{22}$. Les repas festifs, si «frugaux » se revendiquent-ils, reprennent, eux, des éléments des tables compagnonniques ou maçonniques, additionnant ainsi les toasts. Limité aux autorités civiles, judiciaires, militaires, aux fonctionnaires publics, celui qui clôt en l'an VI à Clermont-Ferrand la fête du 18 Fructidor, s'honore d'un coût limité à quatre livres par tête mais n'oublie pas de comprendre «le café et les liqueurs $»^{23}$. Ouvert à 632 citoyens, celui du bourg de Neschers (Puy-de-Dôme), organisé quelques mois avant, le 15 brumaire an VI (5 novembre 1797), à la gloire de Bonaparte et de la paix de Campo-Formio, avait multiplié les toasts : une vingtaine, associant la République, la Constitution de l'an III, l'Égalité, la Justice, le peuple souverain, Bonaparte, Hoche, les morts « au champ d'honneur », les républiquessœurs de Hollande, de Suisse ou d'Italie, les États-Unis, mais tout autant les martyrs de Prairial en la personne de Soubrany, « les patriotes victimes de la réaction de l'an III, de l'an IV et de l'an V », les cultivateurs «néo-jacobins » égorgés par les membres de l'Institut philanthropique cette dernière année, et, en désespoir de cause,... la charrue ${ }^{24}$. Car le banquet peut aussi être un moment de fraternité et d'unité (fût-elle factionnelle), d'abandon des distances et des postures sociales. Le comédien Dorfeuille, un temps président de la Commission de justice populaire de VilleAffranchie, et compagnon de route des représentants Collot d'Herbois et Albitte, sait, au milieu des sans-culottes de l'armée révolutionnaire, donner sens politique à toute scène et théâtraliser les éléments les plus populaires d'une cérémonie commune. À Saint-Étienne, devenue Arme-

\footnotetext{
${ }^{19}$ René BousCAYrol, Les lettres de Miette Tailhand-Romme (1787-1797), Aubière, 1979. Lettres $\mathrm{n}^{\circ} 6$ de fin mai 1788 , p. $28 ; n^{\circ} 16$, du 19 juin 1788 , p. $40 ; n^{\circ} 20$, du 23 juin 1788 , p. $43 ; n^{\circ} 21$, du 24 juin 1788 , p. $44 ; n^{\circ} 25$, du 4 juillet 1788, p. 63 ; n 33, du 3 août 1788, p. $73 ;$ n $^{\circ} 34$, du 4 août 1788, p. 74-75; n 46, de mi-janvier 1789, p. 88.

${ }^{20}$ Ibidem. Lettre ${ }^{\circ} 12$, du 15 juin 1788 , p. 35.

${ }^{21}$ Ibidem. Lettre ${ }^{\circ} 74$, premier trimestre 1791, p. 114.

22 Paul Vergnet (présentation), Mémoires de Barras, Paris, Le Prat, 1946, p. 203.

${ }^{23}$ AD Puy-de-Dôme, L 665 (1). Programme de la fête.

24 AD Puy-de-Dôme, L 663. Procès-verbal de la fête. Cf. Philippe BouRdin, Les «jacobins » du bois de Cros (Clermont-Ferrand, an V) : chronique d'un massacre annoncé », Annales historiques de la Révolution française, $\mathrm{n}^{\circ} 308$, avril-juin 1997, p. 249-304.
} 
Commune, il organise le 25 décembre 1793 un grand charivari déchristianisateur qui fait suite au mariage d'un couple d'indigents, dotés par souscription patriotique. La fête est prolongée par un banquet citoyen, qui se veut geste philanthropique : les officiers municipaux servent les pauvres à table. "Le peuple étoit là dans toute sa pureté originelle; c'étoit la nature, c'étoit la vertu. L'Antiquité n'a rien produit de comparable. Où es-tu, Jean-Jacques ? Tu aurois fait un bon dîner », conclut Dorfeuille, commentateur de ses propres réussites et interprète très libre des plus austères recommandations du philosophe genevois ${ }^{25}$. N'avait-il pas déjà profité, le 9 décembre précédent, d'un repas républicain pour effacer les distinctions de civilité qui lui paraissent relever d'un ancien temps :

« Des patriotes administrateurs et des militaires de tout grade se sont réunis le 19 frimaire pour dîner frugalement. Ils étoient près de 100. La fraternité la plus piquante a régné pendant ce repas vraiment patriote. Des hymnes civiques et une ode récitée avec beaucoup de véhémence ont inspiré un enthousiasme sublime dans l'assemblée. On n'y a pas juré, comme le faisoient les Muscadins, contre l'hôte de la maison et ses filles, quoiqu'ils l'eussent un peu mérité, en oubliant les haricots et les pommes de terre, qui doivent être la base d'un banquet sans-culottique. À la fin du repas, le citoyen Grandmaison, commandant de la gendarmerie, a apporté deux ou trois pintes de vin rouge dans une casserole figurant la coupe de l'égalité. Dorfeuille s'est écrié : voilà le sang des rois, Républicains buvons! C'est ici la conjuration du peuple. Le vase a circulé, et chaque bouche, empressée, avide, croyoit, en buvant cette liqueur, dessécher les veines des tyrans de l'Europe.

Dans cette extase patriotique, tous les convives ont regretté que de pareils festins ne fussent pas plus souvent à l'ordre du jour. Pour établir l'égalité parfaite, quelques-uns ont proposé que les états-majors, que les administrateurs, que tous les fonctionnaires publics mangeassent désormais, chaque jour de décade, à la gamelle. Cette proposition a été généralement applaudie et l'assemblée, par ses acclamations, a témoigné combien elle désiroit la voir réaliser $»^{26}$

\section{La culture des apparences}

Le même souci d'égalité vaut pour le costume. Les gouaches des frères Lesueur sont là pour nous tendre le miroir de ces boutiquiers et maîtres artisans des sections parisiennes ou de leurs épouses, tous amalgamant, autant que leurs moyens le leur permettent, les nouveaux éléments, les nouvelles couleurs de la garde-robe aux pièces plus anciennes de leurs armoires ${ }^{27}$. Les réticences de plus d'un représentant des nouvelles instances politiques à se confondre avec la sans-culotterie sont bien connues, et chacun pense à Robespierre, portant culotte, rayures anglaises et perruque poudrée, ou à la célèbre guerre des cocardes de l'automne 1793. Moins évidemment visible qu'un représentant du peuple, Nicolas Ruault refusera ainsi de coiffer le bonnet rouge, qu'il voit se démultiplier en mars 1792 dans l'enceinte de la société mère des Jacobins, dans les rues et au spectacle, sur le crâne de Dumouriez comme sur celui d'un buste de Voltaire honoré par les comédiens du Français, avant que Pétion n'impose momentanément de remiser le couvre-chef, par crainte de troubles à l'ordre public. Ruault reprend l'argument : «pour moi, je ne l'ai point mis et ne le mettrai point ; rien ne me paraît plus maussade et moins nécessaire : il n'y a guère que la moitié de la société qui se coiffe ainsi. Il est à craindre, si cela dure, que le bonnet ne fasse schisme, entre ceux qui le portent et ceux qui ne veulent pas le porter $»^{28}$. La famille Romme se divise sur ce qui est vu comme des modes, dont le sens politique n'est manifestement pas compris. Que le procureur Gilbert Tailhand affiche jour et nuit une cocarde nationale et son entourage crie au fanatisme quasi religieux ${ }^{29}$. Que Gilbert Romme donne l'exemple d'une volontaire acculturation, et ce sont moqueries rapportées par sa nièce :

\footnotetext{
${ }^{25}$ Bibliothèque municipale de Lyon, fonds Coste, $\mathrm{n}^{\circ} 116256$ (1 et 2). Journal de Ville-Affranchie et des départemens de Rhône et Loire, $\mathrm{n}^{\circ}$ 34, 5 nivôse an II (25 décembre 1793). Lettre de Dorfeuille au rédacteur.

${ }^{26}$ Ibidem, $\mathrm{n}^{\circ} 23,23$ frimaire an II (13 décembre 1793).

${ }^{27}$ Cf. Philippe de CARBonnieres, Lesueur. Gouaches révolutionnaires, Paris, Musée Carnavalet, 2005.

${ }^{28}$ Christiane RIMBAUD et Anne VASSAL (présentation), Nicolas Ruault, op. cit. Lettre du 20 mars 1792, p. 280.

${ }^{29}$ René BousCAYrol, Les lettres de Miette Tailhand-Romme, op. cit. Lettre n ${ }^{\circ}$ 58, mi-août 1789, p. 100 :

«On exige que tout le monde porte la cocarde, les femmes refusent de se soumettre à cet ordre ridicule, ce qui donne lieu à des disputes continuelles. On pousse quelquefois la folie assez loin pour mettre en prison les plus récalcitrants. C'est un fanatisme révolutionnaire qui n'est pas excusable. Ce signe de ralliement est très déplacé sur la tête des femmes. Les paysannes qui le portent ne savent même pas ce que veut dire la réunion des trois couleurs.
} 
« Ma grand'mère se moque de nous. Elle n'entend rien en politique et tourne en ridicule tout ce que nous disons. La mode des sans-culottes a fourni une ample carrière à sa critique. Je conviens qu'elle n'est pas avantageuse. Elle donne l'air commun à tout le monde et particulièrement à $\mathrm{M}$. Romme. Il n'est plus connoissable depuis qu'il a quitté la poudre, pris la veste et les pantalons. Avec ce costume il ressemble assez au cordonnier du coin $»^{30}$.

«L'air commun »? C'est sans doute le plus bel hommage que la jeune fille pouvait rendre à son oncle, mais c'est assez dire le besoin de distinction qui continuait de l'habiter, elle qui adorait créer et coudre la broderie ${ }^{31}$ et avait eu à mesurer en diverses occasions le sens social du vêtement. Ainsi avait-elle observé lors des foires auvergnates de Saint-Gervais, où affluent les montagnards désireux de vendre toiles et bestiaux, les épouses des négociants accompagnent leurs maris et font assaut de leurs toilettes : "Rien n'est ridicule comme des plumes blanches et des robes de soie dans un embarras de foire. Les paysans qui passent à côté de ces belles dames les accrochent pour se venger d'un luxe qui insulte à leur misère ${ }^{32}$. Le conflit est en effet toujours possible : à Riom s'affrontent, pour les Pâques de 1789, jeunes gens de la ville et paysans du bourg voisin de Ménétrol $^{33}$. Bien moins qu'une lutte communautaire ancestrale, comme il en existe des centaines entre villages voisins partout en France, il faut y voir les conséquences d'une différenciation sociale marquée et revendiquée. Elle peut se faire au détriment de la jeune bourgeoise, traitée de haut par $\mathrm{M}^{\mathrm{me}}$ de Capponi, épouse du seigneur du bourg voisin de Combronde : «Elle est aussi fière qu'il est modeste. C'est une créole qui a pris tous les ridicules du nouveau monde. Elle se fait servir par une négresse qui obéit à ses moindres mouvements [...]. Elle ne se sert jamais de ses bras ; on dirait que c'est un automate. Elle ne travaille pas de peur de compromettre sa dignité. Elle a toujours des gants qu'elle ne quitte pas même pour manger ${ }^{34}$. La famille Romme avait été d'autant plus perturbée lorsque Gilbert Romme avait introduit en son sein le jeune comte russe Stroganov : au « sérieux qui glace » et bientôt la subjugue de l'austère précepteur s'ajoutent les modes et les mœurs des cours européennes, même contrôlées par une éducation spartiate :

«Mon amour-propre souffre d'être si simplement vêtue. J'aurais ma robe des dimanches tous les jours que je n'égalerais pas l'élégance de nos étrangers. Ils sont toujours mis comme s’ils étaient à la cour. Mon oncle a des habits de soie de toutes les couleurs, des manchettes et des jabots de points d'Angleterre. Il prend beaucoup soin de sa coiffure. Il est crépé et poudré à blanc. Il lui arrive souvent de se faire peigner deux fois par jour. La toilette du jeune comte donne beaucoup de peine à son valet de chambre. Il est très difficile et d'une propreté minutieuse. Il se lave tous les matins depuis la tête jusqu'aux pieds et change de linge tous les jours. La chemise qu'il quitte est plus blanche que celle que nous prenons le dimanche [...]. Il y a si loin de nos habitudes aux leurs que tout ce qu'ils font nous parait extraordinaire. Cette différence dans leurs usages et dans leurs fortunes me met dans une position gênante ${ }^{35}$.

Miette, qui, sur les conseils sévères de sa grand-mère, cache ses cheveux et sa beauté sous un bonnet de dentelle ( «elle voudrait que je m'enterra comme elle dans une capote ${ }^{36}$ ), aura beau tenter de se convaincre, gagnée aux lumières de son oncle : «L'esprit est une puissance qui en impose plus que les titres et les beaux habits $»^{37}$, elle reviendra souvent sur son infériorité sociale : «La société des riches ne fait pas de bien à ceux qui ne le sont pas $»^{38}$. Elle restera donc bouche bée devant les fourrures russes (pelisses et manchons) distribuées à ses parents, qu'elle essaie en cachette et dont ses cousines, plus fortunées, la jalousent ${ }^{39}$; devant les atours de sa tante de

Mon père qui se met à genou devant tous les hochets de la Révolution a voulu avoir sa cocarde dans son lit; il la porte sur son sein, en façon de scapulaire. Le premier jour qu'elles parurent il en fit chercher beaucoup pour que toute la maison donna l'exemple ».

${ }^{30}$ Ibidem. Lettre $\mathrm{n}^{\circ} 66$, fin août 1790, p. 108.

${ }^{31}$ Ibidem. Lettre ${ }^{\circ}$ 67, mi-septembre 1790, p. 109.

${ }^{32}$ Ibidem. Lettre n ${ }^{\circ} 36$ du 6 août 1788, p. 76.

${ }^{33}$ Ibidem. Lettre n ${ }^{\circ} 52$ d'avril 1789, p. 95.

${ }^{34}$ Ibidem. Lettre $\mathrm{n}^{\circ} 25$ du 4 juillet 1788, p. 50.

${ }^{35}$ Ibidem. Lettre ${ }^{\circ} 11$ du 14 juin 1788, p. 35 .

${ }^{36}$ Ibidem. Lettre de mi-juillet 1788, p. 66.

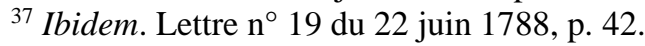

${ }^{38}$ Ibidem. Lettre de mi-juillet 1788, p. 66.

${ }^{39}$ Ibidem. Lettre $\mathrm{n}^{\circ} 22$, du 25 juin 1788, p. 47. 
Rochefort : parure de diamants, robes et corsets faits main, peignoir de mousseline brodée, etc. ${ }^{40}$. Mais, dans la tradition de la civilité dont Miette est l'héritière, les «honnêtes gens », chacun toutefois selon son rang, et par souci de modération chrétienne (en l'occurrence janséniste), sont invités à se détourner du luxe et des ornements. Ce, malgré les tentations d'une presse féminine en plein essor (Journal de la mode et du goût entre 1790 et 1793, Journal des Dames sous le Directoire $^{41}$ ), qui diffuse volontiers les derniers codes parisiens, les meilleures adresses, et le rôle du colportage, des foires et des marchés dans ce que Daniel Roche considère comme une véritable « révolution vestimentaire », à inscrire dans la naissance d'une société de consommation, pour noter aussi : «Au terme de l'Ancien Régime, [...] pour tous les groupes sociaux, sauf les bourgeoisies marchandes et artisanales, la valeur des garde-robes a augmenté beaucoup plus rapidement que celle des biens d'usage, et pour tous sans exception, plus que l'accroissement moyen des patrimoines mobiliers [...]. Les catégories intermédiaires, les domestiques, les plus riches salariés, les bourgeois enrichis conduisent le bal des conquérants du paraître et de l'aisance ». Petits et moyens bourgeois maintiennent dimorphisme sexuel (au profit des parures féminines) et homogénéité du comportement quel que soit le niveau de fortune, dépensant même peut-être relativement un peu moins que leurs ancêtres de la fin du XVII ${ }^{\mathrm{e}}$ siècle - six fois plus cependant que le compagnon ou le manœuvre salarié qu'ils fréquentent quotidiennement; bourgeoisies d'office et de talent augmentent notoirement leurs garde-robes, sans qu'elles pèsent plus lourd qu'autrefois dans les patrimoines mobiliers, sans qu'elles tendent à l'ostentation, sinon celle des accessoires ${ }^{42}$.

$\mathrm{Si}$ les admirables portraits de grandes bourgeoises, parfois anoblies, peints par David entre 1790 et 1792, inaugurent chez l'artiste un style nouveau (modèles représentés aux trois quarts, fonds nus et vifs affirmant la touche du pinceau), s'ils révèlent la libération des corps, débarrassés des robes empesées de l'Ancien Régime, ils ne valorisent pourtant aucunement colliers et bijoux : Mmes Thélusson, Tourteau, Pastoret, Trudaine sont dans les apprêts simples (mais cossus) du quotidien, les trois dernières arborant seulement de larges ceintures vives, bleues ou rouges, selon la mode, sur des ensembles blancs ou noirs rehaussés du blanc des corsages. La pose est beaucoup plus libérée que lorsqu'il peignait le buste en majesté d'Antoine-Gabrielle Charpentier, première femme de Danton: une robe noire au col de dentelle, se confondant presque avec le brun foncé du fond si elle n'était relevée d'un fichu-menteur blanc immaculé (alors très en vogue), une sage coiffe de lingerie fine cachant les cheveux. Lorsque, en 1798, Alexandre Bally fait les Portraits de Monsieur et Madame Cretté, il met encore en valeur la soie couleur de jais de la robe Directoire portée par cette bourgeoise de province, son large bonnet noué par un ruban de satin, qui désormais laisse s'échapper de longues et lourdes mèches. Quant au mari, maître de pension à Orléans et propriétaire d'un cabinet d'histoire naturelle, il persiste à porter perruque poudrée et redingote marron, seul le gilet à rayures vert et orange et le jabot de mousseline orné d'une broche antiquisante sacrifiant au temps présent. Sans perruque ni gilet ostentatoire, Michel Belot, croqué en 1791 par son gendre Martin Drolling en train de lire les réflexions de Mirabeau sur la Constitution civile du clergé, se veut sévère dans son visage comme dans sa mise, une veste de velours brun à larges revers ${ }^{43}$.

Habitués aux couleurs sombres et uniformes, et revendiquant cette sobriété dans ces peintures de commande dont le nombre ne cesse de croître durant la Révolution, manière de s'inscrire dans ce monde en gésine, les membres aisés du tiers état se voient imposer le noir aux États généraux et perçoivent surtout la discrimination qui leur est faite par rapport aux deux autres ordres - il faut attendre un décret du 15 octobre 1789 pour que l'Assemblée ne tolère plus de distinction de

\footnotetext{
${ }^{40}$ Ibidem. Lettre ${ }^{\circ}$ 65, fin août 1790, p. 107

${ }^{41}$ Cf. Anne-Marie KLEINERT, «La mode, miroir de la Révolution française », in Modes et Révolution (1780-1804), catalogue de l'exposition du Musée de la mode et du costume (Palais Galliera), Paris, Éditions Paris-Musées, 1989, p. 59-81 ; Françoise VITTU, «Presse et diffusion des modes françaises », ibidem, p. 129-136.

${ }^{4}$ Daniel Roche, La culture des apparences. Une histoire du vêtement (XVIIe-XVIIIe siècle), Paris, Fayard, 1989, p. 110 et 115 .

${ }^{43} \mathrm{Cf}$. Nicole PELLEGRIN, Les vêtements de la Liberté. Abécédaire des pratiques vestimentaires françaises de 1780 à 1800, Paris, Alinéa, 1989, p. 157 et sqtes.
} 
costume et de rang sur ses bancs, «mesure propre à établir une précieuse confraternité » ${ }^{44}$. Avant que le demi-deuil des privilèges puis le deuil du roi ne la fasse paraître suspecte, la simplicité de la teinte est perçue cependant comme une preuve de patriotisme et devient la norme : Robespierre luimême se fait représenter par Labille-Guiard dans un de ces habits étroits en drap noir uni à la Révolution, que les élégants ne dédaignaient pas à la fin de l'Ancien Régime, quoique les différences sociales en fussent abolies - Calonne selon Vigée-Lebrun (1784), Lavoisier selon David (1788), ne sont pas autrement vêtus ${ }^{45}$. Pour en revenir au juge Pierre Lecocq et à sa famille, le pater familias choisit de poser en manteau noir de juge au tribunal de district d'Arras, où il siège depuis 1790, arborant en sautoir tricolore l'insigne de ses fonctions, et tenant à la main un chapeau noir à plumet du même ton, orné d'une cocarde nationale de belle taille. Un enfant ailé portant bonnet phrygien, figurant le génie de la liberté, tire pudiquement un rideau bleu sur l'habit, le rabat et les armoiries du conseiller royal qu'il fut - «l'ambiguïté réside [...] dans le fait que la toile montre ce que Lecocq prétend cacher » : est-ce là signe de regret ou de modération ? Sa femme comme ses enfants, en tout cas, ont adopté qui dans sa robe ou sa tunique, qui dans ses rubans de cheveux ou de souliers, qui dans sa ceinture, les trois couleurs de la nation ${ }^{46}$. Même démonstration chez Charles Collet, son collègue juge de paix du canton de Lenoncourt en Lorraine : carmagnole et chapeau noirs, la première, concession à la culture des sans-culottes, éclairée par le revers bleu du gilet piqué, le second par la cocarde, le père a fait tailler pour deux de ses fils et sa fille des vêtements de même coupe et de mêmes couleurs, gilets et fines dentelles blanches rappelant l'aisance et le souci de l'hygiène, tandis que le bleu, assorti aux yeux des gamins, l'emporte, et de loin, sur le rouge. L'aîné des garçons, maladroitement mis en avant par le peintre, qui le présente presque en pied tandis que ses parents sont assis, dit la fierté de l'engagement familial : il a revêtu l'habit de garde national, portant fusil et sabre ${ }^{47}$, et tendant, au bout d'un ruban tricolore, une médaille qui résume le combat de toute la maison : « Respect à la loi $»^{48}$.

Le 25 floréal an II (14 mai 1794), conscient de la nécessaire rupture des costumes officiels avec des époques révolues, le Comité de salut public charge pourtant David de les réinventer et de les approprier "aux mœurs et au caractère de la Révolution », avec pour principes l'hygiène garantie par la libre circulation de l'air, la liberté du mouvement, l'égalité qui doit défendre de distinguer la fortune et le rang. L'utopie des lavis qui en résultent, fortement inspirés d'un style gréco-romain et de l'orientalisme, inspirera ultérieurement a minima les tenues officielles du Directoire, destinées à mieux délimiter la sphère du politique - Mérimée, Moreau le Jeune iront alors de leurs propres projets. La question de la symbolique politique du vêtement officiel est pourtant abordée bien avant sur le terrain et sur le vif des événements. Ainsi à Lyon, désormais Ville-Affranchie, dirigée par la Commission temporaire de surveillance républicaine. Les membres de cette dernière ont décidé d'un uniforme : un habit gros bleu avec collet rouge, culotte rouge, auquel les représentants préféreront un costume gris de fer ; une culotte en daim ; un portemanteau de peau et un manteau; un chapeau tricorne avec plumet tricolore; un bonnet de police; des pistolets ; un sabre ; une ceinture à cartouchière et un baudrier noir ; des bottes «à l'américaine » et des éperons bronzés ; un ruban tricolore plissé en sautoir, avec un faisceau et une médaille ; des

\footnotetext{
44 Jean-Marc DEVOCELLE, «D'un costume politique à une politique du costume. Approches théoriques et idéologiques du costume pendant la Révolution française », in Modes et Révolution (1780-1804), catalogue de l'exposition du Musée de la mode et du costume (Palais Galliera), Paris, Éditions Paris-Musées, 1989, p. 83-103.

${ }^{45}$ Pierre Arizzoli-Clementel, «Les arts du décor », in Philippe Bordes et Régis Michel, Aux armes et aux armes. Les arts de la Révolution (1789-1799), Paris, Adam Biro, 1988, p. 305.

${ }^{46}$ Dominique Doncre, Huile sur toile, 0,98 x 0,82 cm, in Philippe Bordes et Alain Chevalier, Catalogue des peintures, sculptures et dessins, Musée de la Révolution, Vizille, 1996, p. 71.

${ }^{47}$ On sait combien les petits Montmorency à coquille, modèles de sabres les plus répandus dans les gardes nationales, inspirèrent les arts décoratifs, bronziers et sculpteurs les ornant pour mieux distinguer leurs propriétaires, nobles ou bourgeois, et la fortune de ceux-ci : emblèmes des trois ordres, feuilles de chêne et de laurier, lion, coq, bonnet, etc. Cf. Riom, la Révolution, ses images, catalogue de l'exposition du Musée Mandet (20 septembre -31 décembre 1989), Riom, 1989.

${ }^{48}$ LAFRAnCE, Charles Collet, juge de paix du canton de Lenoncourt en Lorraine, et quatre de ses enfants, 1793. Miniature sur ivoire, 0,12 x 0,12 cm, Musée de la révolution, Vizille.
} 
gants de peau à l'espagnole ${ }^{49}$. Le registre des comptes de la Commission ne laisse pas d'étonner par la part constante et importante des dépenses vestimentaires. Drap bleu national, dizaines de chapeaux et de plumets de coq, galons, douzaines de bas de coton ou de soie, mouchoirs, décorations, bonnets de poils, culottes, fontes de pistolets, sabres, gants, bottes, sellerie, il y en a pour plus de 14167 livres entre le 7 frimaire et le 11 ventôse an II (27 novembre $1793-1^{\mathrm{er}}$ mars 1794), soit près de la moitié de la subvention prévue pour l'installation de la Commission ! Et la panoplie ne cesse d'être complétée : chaque commissaire, à partir d'une décision du 19 frimaire (9 décembre 1793), dispose d'un "portefeuille à cadenas fermant à serrure », sur lequel est inscrit son nom, et d'un écritoire. L'accoutrement fait-il des envieux ? Des particuliers semblent le copier et provoquent le même jour les plaintes des titulaires légitimes, qui du coup rajoutent encore une ceinture tricolore portée durant les heures de travail... ${ }^{50}$. Se distinguer et distinguer, une nécessité dans un monde perçu hostile ; jusqu'aux autorités élues, qu'il faut contrôler et qui ne peuvent dès lors s'ériger en rivales : aucun officier public ne pourra donc se rendre devant la Commission avec ses insignes nationaux, sans en avoir demandé l'autorisation aux représentants en mission à compter du 25 nivôse (14 janvier 1794) ; aucun particulier ne pourra porter une pièce d'uniforme s'il n'est sous les drapeaux, car l'on craint les rebelles qui se camouflent, à partir du 29 pluviôse (17 février 1794) ${ }^{51}$. Jusqu'aux moustaches abandonnées aux seuls militaires, «considérant que dans une commune où il reste tant de traîtres à découvrir et à punir ", l'intérêt commun exige de prévenir «tous les moyens de déguisement $»^{52}$.

Tyrannie du vêtement et de la mise ? N'en mésestimons pas le rôle symbolique : l'habit est l'un des signes ostentatoires du pouvoir conféré à des hommes pour l'essentiel ignorant de Lyon et de ses réalités, et d'autant plus libres de leurs gestes, désirés exemplaires et repérables. $\mathrm{Ne}$ déclarent-ils pas eux-mêmes aux administrateurs qu'ils contrôlent: "la publicité, magistrats républicains, est la sauvegarde et le garant de la liberté »— sous-entendant que tout acte public, fût-il simple délibération, suppose la présence et la reconnaissance du peuple ${ }^{53}$ ? Cette valeur de l'exemple, théâtrale et morale, fonde pour partie la Terreur, telle que la conçoivent l'ancien comédien Collot d'Herbois, le ci-devant préfet du collège oratorien de Nantes, Fouché, ou l'ami et subordonné de ce dernier, le ci-devant premier vicaire constitutionnel de l'Allier, défroqué, PascalAntoine Grimaud ${ }^{54}$. Il a bien assimilé la leçon, non sans distance ironique ; la joie fébrile qui l'étreint lorsqu'il revêt sa tenue civile officielle est un mélange de satisfaction devant l'abondance offerte, de fierté pour la puissance représentée, à peine tempérée par la peur du qu'en dira-t-on, ainsi exprimée à sa sœur :

« Je ris de bon cœur quand je pense que [...] tu te fais une idée grotesque de mon costume. Dans le vrai, qui eût pu imaginer, il y a quelques années, que je serois en uniforme national un des agens moitié civils moitié militaires de la république françoise? Mais tant il est vrai que les sentimens font tout, je t'assure que je ne me suis pas trouvé étrange à peine un quart d'heure dans mon habillement. Pour te faire rire, représente-toi ton frère en habit de drap bleu foncé, avec un grand colet d'écarlate et le tout de la plus belle qualité, gilet pareillement d'écarlate, une culotte bleue du même drap que l'habit, le tout avec des boutons jaunes nationaux et presque dorés, un magnifique chapeau avec une gance jaune et un superbe panache aux trois couleurs, un sabre à la houzarde et un collier tricolore brodé avec les attributs de la liberté, de l'égalité, quelquefois en culotte de peau, en bottes, en bonnets de discipline ou en bonnets à poil, etc. Riras-tu à ton aise ? Eh bien, je te l'assure, il me semble que je suis né dans cet état, tant je suis identifié avec la chose publique que je servirai jusqu'à la mort de toutes les manières qui seront en mon pouvoir et qui me seront offertes par les circonstances [...]. Il nous a en

\footnotetext{
${ }^{49}$ A.D. Rhône, 3158 . Registre des recettes et dépenses de la Commission temporaire de surveillance républicaine.

${ }^{50}$ A.D. Rhône, 31 1 50. Registre des délibérations de la Commission temporaire de surveillance républicaine. P. v. du 19 frimaire an II (9 décembre 1793). Le 29 frimaire an II (19 décembre 1793), sur proposition du général Parein, il est décidé que seuls les membres de la Commission porteront le collet rouge sur leur habit bleu, tandis que leur secrétaire portera durant ses fonctions un collet bleu.

${ }^{51}$ Ibidem. P. v. du 25 nivôse an II (14 janvier 1794). A.D. Rhône, 311 52. Arrêté de la Commission temporaire du 29 pluviôse an II (17 février 1794).

${ }^{52}$ A.D. Rhône, 31152 . Arrêté de la Commission temporaire du 25 nivôse an II (14 janvier 1794).

${ }^{53}$ B.M. Lyon, Fonds Coste, Ms 676. Adresse de la Commission temporaire au district de la campagne de CommuneAffranchie, 25 frimaire an II (15 décembre 1793).

${ }^{54} \mathrm{Cf}$. Philippe Bourdin, Le noir et le rouge. Itinéraire social, culturel et politique d'un prêtre patriote (1736-1799), Clermont-Ferrand, Presses universitaires Blaise Pascal, 2000.
} 
outre été fait cado d'une douzaine de mouchoirs de poche, de six paires de bas, de six cravates que je fais mettre en col, d'une douzaine de chemises [...], d'une ceinture à faux fouraux avec deux pistolets, d'un portemanteau avec ses courroies, d'une redingote bleue, d'une veste du matin avec des pantalons de drap gris de fer, etc. Tu comprens, ma chère, que ce détail est uniquement pour toi et pour toi seule ; s'il était connu, il ne serviroit qu'à exciter la jalousie contre nous et la malveillance en profiteroit pour déclamer contre la Commission temporaire et les représentans du peuple $»^{55}$.

Celui qui, presque vingt ans auparavant, regardait avec les yeux de l'envie les chasubles de ses confrères, ne peut dans ses fonctions présentes et au milieu des sans-culottes ignorer les risques à s'amuser de colifichets, à vanter le superflu. Frère et compagnon attentif, on le verra pourtant, primesautier, parcourir avec un évident plaisir les rues de la cité des canuts, à la recherche du tablier bleu commandé par sa servante moulinoise, «la Janeton », à laquelle il a déjà envoyé un coupon «aux couleurs nationales » et deux mouchoirs de soie. Que sa sœur lui dise exactement ses désirs, et il fait la «commission avec exactitude». De ces achats fraternels, dressons l'inventaire : un manchon, « un mouchoir de soie très grand pour les jours de décade », un autre à fond gris, deux de linon brodé, un d'indienne à fond blanc «qui sera pour le printemps » et pour un caraco plusieurs aunes de cette même indienne (car « on est fort mal assorti dans ce genre actuellement; c'est pourquoi je n'ai pu choisir à mon aise »), de la dentelle pour deux bonnets, une coiffe «à la mode lyonnoise », trois pièces de lin blanc de différentes largeurs, dont l'une pour border les jupes, du lacet bleu, un cordon de montre tricolore et un chapeau de castor ${ }^{56} .$. Et Pascal-Antoine de regretter que sa cadette ne lui fournisse pas la mesure de son pied «par un fil mince qui marque la longueur et la largeur», puis, une fois sa demande honorée, de courir les boutiques à la recherche d'introuvables babouches : «peines inutiles » et désolantes puisque les souliers « sont devenus bien plus chers qu'à Moulins $»^{57}$. Car, autant qu'un commissionnaire zélé et qu'un consommateur averti, Grimaud est un observateur attentif des tendances vestimentaires. D'où ses remords multiples pour le «manchon bariolé qui est de la grande mode et que le bon marché » lui a fait acheter en négligeant, erreur incompréhensible, la conjoncture politique qui en explique le prix et l'obsolescence brutale :

«Le règne des muscadines est un peu passé ; voilà la cause de la baisse des manchons. J'ai même pensé, mais un peu tard, que ce n'étoit pas le moment de le porter à Moulins ; tu en jugeras toi-même sur les lieux. Au surplus, tu es au-dessus de ces misères. Si il venoit à ombrager les véritables républicains, tu n'hésiterois pas à en faire le sacrifice $»^{58}$.

Contre les négligés des sans-culottes, la réserve des patriotes, l'uniforme des commissaires de la République, Muscadins et Jeunesse dorée - cette « voyoucratie » définie par sa réaction violente à la Terreur, par le jeune âge de ses membres, par leur appartenance entre autres à la petite bourgeoisie à talents et du commerce de luxe, aux réquisitionnaires ${ }^{59}$-, affichent «l'orgueil de leurs élégances ${ }^{60}$, associant passion antiquisante et anglomanie, exagération et dérision. Bosio, Isabey, Vernet en ont gravé des portraits bien connus. Tresses, collets verts ou noirs et cravate dite « écrouélique » (cornet de mousseline mouchetée de rouille dont émerge à peine le visage), souliers pointus, flot de rubans, bas chinés, linge éclatant et verbe haut, prononciation travaillée, tous s'enorgueillissent du contrôle privé de leur apparence, à rebours des modes officielles... mais aussi des symboles nationaux. L'habit vert bouteille ou couleur de crottin » compte dix-sept boutons de nacre pour rappeler l'orphelin du Temple, le col de velours noir se veut hommage au défunt roi,

\footnotetext{
55 Pierre Flament, Lettres inédites de Pascal-antoine Grimaud, vicaire épiscopal de l'Allier, membre de la Commission temporaire de Lyon (décembre 1793-mai 1794), Moulins, 1911. Lettre du 24 nivôse an II (13 janvier 1794).

${ }^{56}$ Ibidem. Lettres des 24 frimaire, 13, 18, 26 nivôse, 21 pluviôse an II (14 décembre 1793, 2, 7, 15 janvier, 9 février 1794).

${ }^{57}$ Ibidem. Lettre du 13 nivôse an II (2 janvier 1793) et lettre du même mois, non datée.

${ }^{58}$ Ibidem. Lettre du 24 frimaire an II (14 décembre 1793).

${ }^{59}$ Nicole Pellegrin, op. cit., p. 106 ; François GENDRON, La jeunesse dorée. Épisodes de la Révolution française, Sillery, Presses universitaires du Québec, 1979, p. 13 et sqtes.

${ }^{60}$ Daniel Roche, «Apparences révolutionnaires ou révolution des apparences », in Modes et Révolution (1780-1804), catalogue de l'exposition du Musée de la mode et du costume (Palais Galliera), Paris, Éditions Paris-Musées, 1989, p. 105-127.
} 
tandis que le gourdin plombé, qui parfois renferme un stylet, serré par une main blanchie à la pâte d'amande, devient l'antithèse de la pique populaire. Les «nudités gazées » des Me'veilleuses aux hautes perruques blondes accompagnent ce monde dont la masculinité est d'abord affirmée par la violence et une forte odeur de musc... Cette liberté des mœurs, éprouvée publiquement (dans les bals, les fêtes et les thés), cette impudence de l'argent étalé par des nouveaux riches sortis d'un monde interlope, suscite au demeurant nombre de satires et de réprobations. Ainsi en l'an VIII chez Pinière, pourfendant la décennie écoulée pour mieux exalter Bonaparte :

«Dignes de tels époux, sous de pompeux habits,
Leurs femmes, s'écrasant et d'or et de rubis,
Ornant leurs fronts hâlés de perruques énormes,
De leurs charmes épais mettant à nu les formes,
Dans ces lieux autrefois à Terpsicore ouverts,
S'amusent un moment de leurs plaisans travers ;
De leur grosse gaîté font retentir les voûtes [...]
Et, portant au Salon le ton de l'antichambre,
Exhaltant, à-la-fois, des vapeurs d'ail et d'ambre,
Dans leur danse pesante ou leur luxe odieux,
Elles révoltent l'âme et fatiguent les yeux ${ }^{61}$.

Sans doute le temps des Inc'oyables est-il fort bref et leur influence sociale strictement limitée. Mais il induit une extrême versatilité dans la culture des apparences que le Directoire, quels que soient ses projets d'habits nationaux, ne remettra jamais en cause. Le vêtement est plus que jamais le reflet des distinctions de tous ordres (politiques, morales, économiques, géographiques), y compris au sein d'une bourgeoisie dont se détachent par leur outrance et leur affichage nombre de parvenus, agioteurs et pourvoyeurs notamment. Ce désaveu de l'habit égalitaire, tel que le souhaitaient les sans-culottes, est aussi une remise en cause de la Révolution, une manière d'affirmer qu'elle est, quoi qu'en disent ses thuriféraires, bel et bien achevée. Plus encore, et il faut replacer ce fait dans un contexte intellectuel général de remise en cause des idéaux et des réalisations révolutionnaires, l'attaque est portée contre la sensibilité des Lumières. L'identité olfactive du Muscadin est choisie à dessein, en rupture avec les légèretés florales appréciées des élites éclairées, quelles que soient les contradictions sociales qui en découlent. On sait combien partie de la médecine de la fin du XVIIIe siècle théorise sur le diagnostic sexuel, géographique et social permis par la bonne interprétation de l'odeur de l'individu, distinguant notamment le corps du pauvre (ainsi Haller, en 1769, dans ses Élémens de physiologie, ou Bordeu, en 1775, dans ses Recherches sur les maladies chroniques). Au nom de l'hygiène corporelle, de la pudeur nouvelle, et du nécessaire démarquage par rapport à l'homme de peine, l'odeur offusquante des parfums trop lourds, trop musqués, qui révèle le corps et l'animal plus qu'elle ne le cache, a été remplacée par un florilège de parfums, savons et pâtes aux fragrances florales ou fruitées. Eau de rose et pâte d'iris parfument ainsi la bouche. Flacons, cotons imbibés, sachets de poudre camouflés dans le déshabillé, assurent la présence discrète mais constante de ces parfums au plus près du corps, boîtes et corbeilles de senteurs, savants pots-pourris poursuivant dans les appartements cette quête olfactive mise en musique par Jean-Philippe Rameau dans sa Gavotte pour les fleurs. Tandis que les élégantes cultivent giroflée et basilic en pot, la nature exulte jusque dans les imprimés des vêtements, les motifs des papiers peints - parures et guirlandes de fleurs, vases ou paniers débordants de bouquets de jacinthes, de muguet, de liserons, de renoncules, de jasmin, de jonquilles et surtout de violettes, de plus en plus finement dessinés sous le règne de Louis $\mathrm{XVI}^{62}$. Tout est bon pour se défaire des miasmes urbains réputés corrupteurs. "L'unité entre la nature et l'homme, note Robert Mauzi, donne l'illusion d'une unité intérieure à l'homme. La sensation restaure entre le cœur

\footnotetext{
${ }^{61}$ BNF, Ye 30 125, Le Siècle. Cf. Philippe Bourdin, «Entre deux siècles, l'impossible bilan : la Révolution au crible de la satire littéraire », in Michel BIARD, Terminée la Révolution..., actes du IVe colloque européen de Calais, $n^{\circ}$ horssérie des Amis du Vieux Calais, 2002, p. 25-42.

${ }^{62}$ Cf. Alain CORBIN, Le miasme et la jonquille. L'odorat et l'imaginaire social (XVIII ${ }^{e}$-XIX ${ }^{e}$ siècles), Paris, Aubier, 1982.
} 
et l'esprit le fil qui s'était brisé. Un simple parfum devient une prise de conscience de soi. Celle-ci a pour effet d'associer au moi la nature, jusque là étrangère ${ }^{63}$.

Étudiant les motifs imprimés des papiers Réveillon, Chrisine Velut remarque cependant : « Dès les années 1790, se traduit aussi dans les papiers peints le « retour à l'antique » sous la forme d'une nouvelle gamme de motifs à la mode, et il est intéressant de noter - tendance qui se poursuit au début du XIXe siècle - un net recul des fleurs dans la hiérarchie décorative ${ }^{64}$. Les références aux modèles de Sparte et de Rome, jusque dans le décors des hauts lieux de la politique, telle la salle de la Convention nationale aux Tuileries, l'impose sans doute, quoique la tribune présidentielle de l'Assemblée soit ornée du «papier peint égalitaire », reproduisant la Déclaration des droits de l'homme et la Constitution de $1793^{65} \ldots$ Le mobilier montré lors de la récente exposition du Musée Carnavalet, Au temps des merveilleuses. La société parisienne sous le Directoire et le Consulat, confirme cette évolution, encore qu'il provienne essentiellement des collections Tallien, Beauharnais, Récamier, et révèle donc à ce titre le goût particulier des séductrices enrichies, contrôlant partie de la vie publique en des espaces privés dont le décor évoque volontiers, comme un miroir tendu, les amours de Psyché, Flore ou Danaé avec Amour, Zéphyr ou Zeus ${ }^{66} \ldots$ Il n'empêche : l'engouement chez certains perdure. Il n'est qu'à observer l'intérieur du juge Pierre Lecocq, dont le salon, est décoré de panneaux de papier peint en arabesques combinant guirlandes florales et scènes mythologiques ${ }^{67}$. L'ancien Conventionnel Bancal des Issarts, rendu à ses terres, des biens nationaux qu'il administre de loin avec le détachement que lui imposent des ressources financières incertaines, nous en administre une autre preuve, donnant depuis Paris ses ordres à sa famille restée en Auvergne : que l'on décore donc, le Directoire finissant, son logement d'un papier « en fleurs simples sans toile dont le dessin seroit agréable ayant quelques petits œillets ou autres petites fleurs légères, fond blanc ou blanc cendré, et les dessins variés de couleur rouge et lillas et vertes [...]. On pourrait accompagner le papier d'une hauteur d'appui peinte sans huile de fond blanc ou blanc cendré ou bleu de ciel, avec des filures couleur gris de vin ou gris cendré ${ }^{68} \ldots$

\section{Les loisirs des amateurs}

Le désir, que la Révolution rend d'autant plus conscient qu'elle en démultiplie les attentes et les attendus, de se placer sur la scène sociale et politique par le costume et le décor privé a pour corollaire un rapport particulier à la représentation artistique - et un goût certain pour le portrait. Dans quelle mesure et dans quels buts le bourgeois est-il amateur, pratique-t-il ou collectionne-t-il ? L'accès à l'art, à l'heure de la Révolution, peut laisser à certains quelque espoir : la demande baisse, les acheteurs les moins fortunés renonçant ou la ruine des courtisans limitant leurs possibilités ; l'émigration ou les emprisonnements vont accroître ces tendances ; l'offre ne cesse d'augmenter. Le prix des œuvres, qui sont de moins en moins dues aux anciens académiciens et privilégient portraits, peintures de genre, allégories antiquisantes, chute faute de clientèle ${ }^{69}$. Les nouveaux riches en profitent pour se passer quelques caprices, fût-ce en traitant comme laquais des artistes renommés. La vengeance de Girodet, en butte aux humeurs de la citoyenne Lange, est exemplaire et de l'orgueil blessé d'un amant éconduit et de la critique sociale et morale d'un peintre depuis peu

\footnotetext{
${ }^{63}$ Robert MAUZI, L'idée de bonheur au XVIII siècle, Paris, 1960, p.

${ }^{64}$ Christine VELUT, La rose et l'orchidée. Les usages sociaux et symboliques des fleurs à Paris au XVIII ${ }^{e}$ siècle, Paris, Larousse, 1993, p. 189.

${ }^{65}$ Pierre ARIZZOLI-ClEMENTEL, «Les arts du décor », article cité.

${ }^{66}$ Au temps des merveilleuses. La société parisienne sous le Directoire et le Consulat, 9 mars-17 juin 2005. Catalogue édité par Paris-Musées, p. 85 et sqtes ; Anne LAFONT, «Á la recherche d'une iconographie incroyable et merveilleuse : les panneaux décoratifs sous le Directoire », Annales historiques de la Révolution française, ${ }^{\circ} 340$, avril-juin 2005, p. 5-21.

${ }^{67}$ Dominique DONCRE, Pierre Lecocq et sa famille, huile sur toile, 0,98 x 0,82 cm, Musée de la Révolution, Vizille.

68 AD Puy-de-Dôme, 2 E 276 (25). Lettre du 15 thermidor an VII (2 août 1799). Cf. Philippe BouRDIN, «Bancal des Issarts, militant, député et notable : de l'utopie politique à l'ordre moral », Revue historique, n 4- 2000, p. 895-937.

${ }^{69}$ Udolpho van de SANDT, «La peinture : situation et enjeux », in Jean-Claude BONNET (sous la direction de), $L a$ Carmagnole des Muses. L'homme de lettres et l'artiste dans la Révolution, Paris, Armand Colin, 1988, p. 333-357.
} 
reconnu : sa Mademoiselle Lange en Danaé, accrochée au Salon de l'an VII, représente l'actrice enrichie dans la provocation de son plus simple appareil, sur le lit grossier de l'usage duquel elle obtint la fortune qui tombe en pluie d'or, sous l'œil d'un énorme dindon figurant l'affairiste qui l'entretient, etc. ${ }^{70}$. Le Dictionnaire des amateurs français au XVIII siècle, publié par Émile Bonnafé en 1884, nous permet une première approche statistique du monde des collectionneurs. À partir de quatre-vingts sources (archives, récits de voyage, mémoires, catalogues, journaux intimes, correspondances, traités, dictionnaires, guides, etc.), l'auteur a recensé 846 collections : $21 \%$ de leurs propriétaires appartiennent à l'aristocratie, $29 \%$ au monde des négociants-fabricants, $20 \%$ à celui des robins, $9 \%$ aux professions libérales, $7 \%$ au milieu des financiers, $14 \%$ au clergé. En moyenne, $42 \%$ des collections comptent des tableaux, particulièrement celles des financiers (à $82 \%)$, des aristocrates $(57 \%)$ et de la robe $(44 \%)$; le clergé $(36 \%)$, le négoce $(29 \%)$ et les professions libérales (24\%) n'en possèdent que plus marginalement, eu égard à l'investissement financier ${ }^{71}$. Dans la deuxième moitié du $\mathrm{XVIII}^{\mathrm{e}}$ siècle, 320 livres en moyenne sont en effet nécessaires pour acheter une peinture dont le thème porte sur l'histoire profane, 310 pour un exemple d'histoire sainte, 230 pour un portrait, 160 pour un paysage, 80 pour une nature morte et, au bas de l'échelle des valeurs, 70 pour une copie «à la manière de ». C'est pourquoi l'on trouvera parmi les propriétaires parisiens de tableaux, avant les magistrats et les avocats, des marchands de vêtements, de produits de luxe, d'alimentation ${ }^{72}$. Plus généralement, sur l'ensemble des objets collectionnés, l'on notera l'éclectisme de la noblesse chez laquelle les peintures voisinent avec les meubles de luxe, les bijoux et les pierres précieuses, les livres rares; l'érudition des gens de robe privilégiant les livres, les antiques, les pierres gravées, les médailles; la tendance du clergé à accumuler des estampes, meilleur marché que les tableaux et riches en sujets religieux ${ }^{73}$. Nul, quel que soit son camp, ne néglige l'engouement pour la caricature, porté par des prix bon marché : 10 à 15 sous la pièce contre 10 à 30 sous pour les gravures des œuvres de David, dix fois plus pour le reste de la production gravée, quand le petit artisan parisien gagne journellement 20 à 50 sous. Comme l'estampe avait trouvé à partir de la deuxième moitié du dix-huitième siècle ses acheteurs dans la petite noblesse et les classes moyennes intellectuelles d'officiers et de conseillers, «la caricature, trop sophistiquée et suspecte peut-être de frivolité, inutile au combat des classes populaires, trouva son public à la fois dans la classe aisée et cultivée de la bourgeoisie (lorsqu'elle n'était point trop puritaine) et dans la fraction révoltée de la noblesse, familière de l'art, provocatrice à l'égard de la monarchie absolue $»^{74}$.

Effet de mode ou véritable engouement créé par la diffusion parisienne de la gravure (plus de 70000 sont produites durant tout le XVIII siècle), les Salons des dernières années de l'Ancien Régime, désirés par le pouvoir, ouverts à une nation qui doit pouvoir admirer les œuvres produites pour la gloire du roi par privilège de l'Académie, accueillent 60000 visiteurs de tous âges et de tous sexes (comme nous le rappellent les représentations peintes ou dessinées de l'époque), flâneurs, curieux, public cultivé, dont le nombre diminue de moitié avec les événements révolutionnaires. Le Salon est accompagné de catalogues, de critiques savantes, mais aussi de pamphlets, usant parfois du parler « poissard ». Ils contribuent à en faire une manifestation «populaire », aux dires de LouisSébastien Mercier, et rendent compte avec dérision de la promenade des pédants et des coquettes, parfois pour mieux fustiger la démocratisation du goût et la représentation peinte du quotidien de la

\footnotetext{
70 Sylvain BeLlenger (dir.), Girodet (1767-1824), catalogue de l'esposition du Grand Palais (23 septembre 2005 2 janvier 2006), Paris, Musée du Louvre éditions, 2005, p. 272-281, « Le tableau d'une vengeance ».

${ }^{71}$ Selon l'étude d'Émile BonNAFE, sur les 846 collections étudiées, 57 relèvent du monde de la finance, 175 de

l'aristocratie, 167 de la robe, 118 du clergé, 250 du négoce et de l'artisanat, 79 des professions libérales.

${ }^{72}$ Georges WiLDENSTEIN, «Le goût de la peinture dans le cercle de la bourgeoisie parisienne au début du règne de Louis XVIII », Gazette des Beaux-Arts, septembre 1950.

${ }^{73}$ Nathalie HeINICH, Du peintre à l'artiste. Artisans et académiciens à l'âge classique, Paris, Éditions de Minuit, 1993 , p. 55 .

${ }^{74}$ Michel Melot, « Caricature et Révolution. La situation française en 1789 », in Politique et polémique. La caricature française et la Révolution, catalogue de l'exposition de l'Université de Californie, de la Bibliothèque nationale de France, et du Musée de la Révolution (Vizille), University of California, 1988, p. 25-32.
} 
bourgeoisie, qui peut favoriser une prise de conscience de classe ${ }^{75}$. On sait combien l'ensemble du tissu social du tiers-état, depuis la première moitié du XVIII siècle, est susceptible de fournir des apprentis-artistes. Mireille Rambaud a dépouillé sur ces cinquante ans cent trente-six contrats d'apprentissage (six architectes, trente-six peintres, trente-neuf sculpteurs, quinze peintressculpteurs, quarante graveurs) : douze apprentis sont issus du monde des ouvriers ou des employés, quatre-vingt-six du milieu des artisans-commerçants, trente-trois de la bourgeoisie (robinocratie, professions libérales et publiques) ${ }^{76}$. La Révolution à l'évidence favorise un changement d'échelle du monde des amateurs et, rompant les digues de la reconnaissance académique, une liberté qui installe davantage de flou sur la frontière avec le monde professionnel. La pédagogie des créations artistiques n'en progresse pas moins. L'architecte de Wailly a ainsi fondé en 1790 la Société des Amis des Arts, montant des expositions et assurant la vente des tableaux par un système d'actions : leur émission a pour objet la constitution d'un capital, utilisé pour acquérir de nouvelles productions qui, par tirage au sort, seront réparties entre les sociétaires. Bref, un système autogestionnaire qui associe collectionneurs, praticiens et simples amateurs ${ }^{77}$. Pour les artistes se pose plus vivement que jamais le problème de l'intelligibilité de leur œuvre : il n'est pas fortuit que paraisse en 1791 une brochure, Essai sur la méthode à employer pour juger les ouvrages des beaux-arts, qui propose entre autres une analyse du Serment du Jeu de Paume de David, en distinguant rigoureusement les différents niveaux de lecture idéologique - le peintre a exposé un premier dessin de l'œuvre dans son atelier en mai et juin de la même année, recevant ses confrères et des amateurs, puis l'a proposé au Salon de septembre, obtenant que le Trésor public prenne l'œuvre à sa charge après l'échec d'une souscription ${ }^{78}$.

Que la compréhension de l'œuvre d'art puisse passer par la lecture de l'imprimé induit évidemment une réduction du public visé. Or, l'on sait combien, grâce notamment aux travaux d'Albert Labarre, Henri-Jean Martin, Daniel Roche et Roger Chartier, combien la possession du livre ne se réduit pas à un cénacle savant et privilégié mais, dans les grandes villes particulièrement, a gagné en un siècle les différentes strates du tiers-état. Dans les années 1650 à Paris, le seuil des cent ouvrages était rarement atteint par les négociants et les bourgeois, tandis qu'il est franchi une fois sur deux par les gens de robe. Un siècle plus tard, la présence du livre s'est affirmée dans toutes les couches de la population parisienne. Les négociants, en plus des ouvrages religieux, des traités de commerce et autres écrits spécialisés, s'intéressent à la poésie, au roman, à la philosophie et veulent rivaliser avec la culture des Académiciens qui ne les reconnaissent pas. En 1780, les plus aisés des domestiques disposent d'en moyenne vingt-huit livres, les plus aisés des salariés de vingtquatre. Dans les villes de l'Ouest du pays, la possession du livre augmente aussi globalement et les collections s'étoffent, certaines riches des titres de la Bibliothèque bleue, " conformes aux capacités de lecture des acheteurs potentiels » selon R. Chartier. Comment ignorer, enfin, la prolifération sous leurs diverses formes des cabinets de lecture ? Ceux que les libraires rattachent à leurs boutiques chez Guerlache à Metz, chez ses confrères à Montpellier, Blois, Chartres, Clermont-Ferrand, Lunéville, Nîmes, Niort ou Pau. Ceux qu'ouvrent les sociétés littéraires, à Millau ou à Mortain, pour créer un espace de convivialité et une fréquentation régulière de leurs membres. Á Nantes, c'est une chambre de lecture que nous décrit Arthur Young, divisée en trois espaces : une bibliothèque, un salon pour lire, un autre pour converser. Elle a été fondée en 1759 par cent vingtcinq associés, payant un droit d'entrée de trois livres après avoir été cooptés et un abonnement annuel de vingt-quatre livres. L'institution est administrée par des commissaires élus, qui se chargent de commandes de périodiques et d'ouvrages, avec une prédilection pour le commerce, la marine, les arts, la littérature. De semblables formules sont attestées à Rennes, au Mans et à Brest,

\footnotetext{
75 Thomas CRow, La peinture et son public à Paris au XVIII e siècle, Paris, Macula, 2000, p. 109 et 115. En 1781, la Rafle de sept ou réponse aux critiques du Sallon considère ainsi que « la halle et les boulevards se sont réunis pour inspirer la plupart des auteurs qui ont écrit avec la délicatesse et le goût ordinaires à ces deux endroits ».

${ }^{76}$ Mireille RAMBAUd, Documents du minutier central concernant l'histoire de l'art (1700-1750), Paris, Imprimerie nationale, 1964, tome I, p. 375-392, et tome II, p. 572-581.

77 Annie BECQ, « Artistes et marchés », ibidem, p. 81-96.

${ }^{78}$ Cf. Philippe Bordes, Le Serment du Jeu de Paume de Jacques-Louis David, Paris, Éditions de la Réunion des Musées nationaux, 1983.
} 
preuve du succès nantais — à Nantes même, six chambres de lecture existeront en $1793^{79}$. Car la Révolution, qui s'empare des bibliothèques des couvents ou des émigrés pour nourrir partie des fonds publics, n'interrompt en rien cette soif de lecture. Elle l'augmente au contraire par l'efflorescence des périodiques, des affiches, lus publiquement dans la rue comme dans les clubs ou dans l'armée ${ }^{80}$. Lire n'est plus un marqueur exclusif du loisir aristocratique ou bourgeois ; réservé au for privé, conduisant à la bibliophilie, l'acte le redevient sans doute.

Avec la bibliothèque de Gilbert Romme, membre du Comité d'instruction publique sous 1'Assemblée législateur et la Convention, auteur majeur du calendrier révolutionnaire, ou les livres de son neveu Jean-Baptiste Tailhand, continue ainsi de fonctionner une mise en réseau des achats et des curiosités, une circulation continue des ouvrages au profit d'une bourgeoise provinciale. La chance a voulu que les Archives départementales du Puy-de-Dôme aient conservé la trace des lectures de toute une partie des élites riomoises entre l'an V et l'an IX de la République, à savoir les 143 emprunteurs qui ont régulièrement fréquenté les bibliothèques Romme et Tailhand, 120 hommes et 23 femmes repérés dans les soixante-sept pages manuscrites qui répertorient plus de quatre cents titres, soit plus d'un millier de volumes échangés ${ }^{81}$. C'est la production littéraire, philosophique, scientifique, historique ou juridique récente qui intéresse au premier chef, avec l'empreinte forte de la deuxième génération des Lumières : pour les 292 ouvrages en circulation dont nous connaissons la date de publication, 81,4\% ont été édités après 1770, $31 \%$ durant la décennie révolutionnaire, périodes durant lesquelles Romme a fait l'essentiel de ses achats, soit directement soit par le biais de ses amis, notamment dans les années 1780 où, précepteur du comte Stroganov, il a les liquidités nécessaires à ses acquisitions. Dans la décennie suivante, durant une partie de laquelle il occupe les fonctions que l'on sait, l'enrichissement du fonds familial n'est pas seulement dû à ses propres curiosités mais aussi à celles qu'il a transmises à son neveu, JeanBaptiste Tailhand. Et, de ce point de vue, plusieurs des livres possédés et prêtés prouvent combien la bourgeoisie riomoise, tout en lisant la Décade philosophique, continue de s'interroger a posteriori sur les choix du gouvernement révolutionnaire. En effet, hormis La Vie de Turgot, De l'importance des opinions religieuses, ou le Plan de législation criminelle, élaboré par Marat (1790), place est faite aux discours de Mirabeau, aux Annales politiques, civiles et littéraires de Simon Linguet et surtout aux anciens Girondins : les Mémoires de Manon Roland, les Mémoires d'un détenu pour servir à l'histoire de la tyrannie de Robespierre, par Riouffe, les Notices pour l'histoire et le récit de mes périls depuis le 31 mai 1793, de Louvet de Couvray. La moitié des titres et des volumes prêtés, quel que soit le sexe des lecteurs, se rapportent à la littérature ancienne et moderne, l'histoire, la géographie et les voyages, ce qui n'empêche pas un retour aux grands classiques fréquentés depuis le collège et à leurs commentaires. De la même façon, l'histoire est appréciée à travers Plutarque, Thucydide ou Tacite, comme à travers Volney. La religion n'est guère prisée, la philosophie davantage mais sans excès et en tout cas guère plus que les traités d'éducation, de morale et de civilité, avec toutefois une appétence particulière pour Machiavel, l'abbé Raynal, Condorcet et les écrits de Kant, lus sitôt publiés.

La plupart des lecteurs (plus du tiers se contentent de moins de cinq volumes, $60 \%$ de moins de neuf) habitent Riom, mais aussi des villages et des bourgs où la famille Tailhand-Romme entretient un réseau de sociabilité, ne serait-ce qu'à l'occasion de sa résidence et ses voyages estivaux. Peu de livres suscitent l'engouement de plusieurs lecteurs et, s'ils le font, traduisent essentiellement les modes littéraires constatées dans la capitale : 273 ne donnent lieu qu'à un seul prêt ; 3 à 6 - Paul et Virginie, de Bernardin de Saint-Pierre, les CEuvres de Florian, La Vie de Catherine II, de Castéra -, et 4 à 7 - Les Soirées littéraires, de Coupé, Vie et amours du chevalier de

\footnotetext{
${ }^{79}$ Roger CHARTIER, Lectures et lecteurs dans la France d'Ancien Régime, Paris, Seuil, 1987.

${ }^{80}$ Françoise PARENT, «La lecture publique sous la Révolution », in Roger CHARTIER et Henri-Jean MARTIN, Histoire de l'édition française, tome II : «Le livre triomphant (1660-1830) », Paris, Fayard-Cercle de la Librairie, 1990, p. 801808.

81 Philippe Bourdin, «La postérité de la bibliothèque Romme : le prêt privé dans les milieux «néo-jacobins » provinciaux », in Philippe BoURDIN, Jean-Luc CHAPPEY, Réseaux et sociabilité littéraires en Révolution, Paris, Société des Études Robespierristes, 2005.
} 
Faublas, de Louvet de Couvray, La Religieuse, de Diderot, les Leçons élémentaires d'arithmétique, de Mauduit. Sur les 107 hommes adultes qui empruntent, 63 ont pour l'heure été identifiés : 18 relèvent des métiers de justice, 10 sont ou ont été des administrateurs élus à différentes fonctions depuis les débuts de la Révolution - avec une implication de la plupart dans la société populaire de l'an II, ce qui n'exclut pas la présence de deux officiers municipaux épurés au 18 Fructidor -, 10 sont des prêtres constitutionnels, 5 des fonctionnaires, 5 des marchands-artisans (perruquier et orfèvres notamment), 4 des médecins, 4 des militaires (dont le général Chapsal, lié avant la Révolution à Dubreul au sein du Salon littéraire, et Croizier, futur aide de camp de Bonaparte), 4 des rentiers, 2 des enseignants. Plusieurs ont entretenu une correspondance suivie avec le défunt Conventionnel, compte parmi ses collatéraux ou ses amis de longue date. Les plus jeunes ont participé à la Société dramatique de Riom, où ils ont accompagné Jean-Baptiste Tailhand pour ses débuts sur les planches. D'autres enfin, administrateurs de différents districts du département, ont eu un engagement montagnard sans ambages et se retrouvent dans les cercles constitutionnels du Directoire.

Une fois parcourus les salons et les cabinets des collectionneurs, passés les moments de lecture, demeurent d'autres temps de loisirs dont les qualités strictement bourgeoises font débat. On a beaucoup glosé, et les frères Goncourt n'y sont pas pour rien, sur les divertissements dansés qui, dans le Paris du Directoire, animent quotidiennement des dizaines de lieux. «Dix-huit cents bals ouverts tous les jours » annoncée par force placards, note Mercier dans son Nouveau Paris (chapitre LXXXIII), qui nous décrit une dansomanie frénétique dans laquelle se complaisent jusqu'aux familles des anciens guillotinés ou qui amène les amateurs à un perfectionnement parfois digne des professionnels : " chaque classe a sa société dansante, et du petit au grand, c'est-à-dire du riche au pauvre, tout danse » (chapitre XCII). Avant que les bals de la cour impériale ne deviennent des spectacles à part entière, des artistes de l'Opéra, Abraham ou Vestris, habitué du salon Récamier, popularisent la gavotte ${ }^{82}$; la valse, importée d'Allemagne, fait concurrence à la contredanse, faisant la réputation des jardins de Tivoli, de l'Élysée, de Bagatelle, etc. La province n'est pas à l'écart de cette mode, qui favorise joutes amoureuses ou libertines, ni des distinctions symboliques de la stratification sociale de l'ancien tiers-état. Elle trouve volontiers l'occasion d'expérimenter les dernières lubies de la capitale et de mesurer déjà le fossé des générations, comme Miette Tailhand nous le rapporte à l'occasion d'une sortie clandestine puis du mariage de son frère :

«J'ai fait une farce de carnaval, [...] qui aurait pu me compromettre et qui ne m'a pas amusé. Depuis longtems je désirais connaître un grand bal. Pour contenter mon envie, j'imaginai de me déguiser en paysanne et d'aller, avec la domestique de ma mère, la chercher chez Madame Boutarelle [Boutarel, épouse d'un homme de loi riomois, N.A.] où elle dansait. Je fis part de mon projet à Mablotte qui est de mon âge et à peu près de ma taille. Elle me prêta sa robe des dimanches [...]. Les domestiques attendaient leurs maîtresses dans une antichambre qui précède la salle de danse. Par le moyen d'une porte vitrée, elles pouvaient voir ce qui se passait dans le bal. Je me plaçai dans l'endroit le plus obscur de peur d'être reconnue. Les yeux fixés sur les danseurs je ne perdais pas un de leurs mouvements. Les jeunes qui entraient et qui sortaient faisaient aux domestiques des plaisanteries plus malhonnêtes les unes que les autres. Le plus libertin de tous s'écria en me voyant : «Voilà du fruit nouveau ». Il s'approcha de moi, me passa la main sous le menton et me dit mille impertinences. Je n'osais ni le regarder ni lui répondre de peur de me trahir. Nanette le fit pour moi. Elle le repoussa si vigoureusement qu'il eut pas envie d'y revenir ${ }^{83}$.

« La danse a ses modes comme la toilette. Celle d'aujourd'hui est vraiment ridicule. Il faut être bien jeune pour la suivre. Madame Teillot qui vient de Paris nous a servi de modèle. Le dos courbé, formant un demi-cercle, les bras pendants, la tête penchée, un laisser-aller général qui ne manque pas de grâce. L'élégante dame qui nous donnait le ton a bientôt eu des imitateurs. Sans s'en apercevoir on prend les habitudes des personnes qu'on admire.

Á la seconde contredanse tous les danseurs ont suivi l'exemple de Madame Teillot, excepté nos mères qui ont conservé leur port majestueux. Elles ont critiqué notre mauvais goût, et nous leur danse antique. La jeunesse aime les innovations et saisit avec plaisir tous les changements. Une réforme dans la danse a fait discuter comme

\footnotetext{
${ }^{82}$ Françoise DARTOIS, «Les spectacles dansés pendant la Révolution et l'Empire », in Philippe BouRDIN et Gérard LOUBINOUX, Les arts de la scène et la Révolution française, Clermont-Ferrand-Vizille, Presses universitaires Blaise Pascal, 2004, p. 439-484.

${ }^{83}$ René BousCayrol, Les lettres de Miette-Tailhand-Romme (1787-1797), op. cit., Lettre ${ }^{\circ} 43$ de début janvier 1789.
} 
s'il avait été question d'une affaire d'État. Les opinions étaient partagées, on délibérait sérieusement sur un sujet qui n'aurait dû prêter qu'à rire $»^{84}$.

Mais c'est sans doute plus nettement encore dans le théâtre, lieu de croisement des sociabilités, que se manifeste ce que la critique dénoncera bientôt comme un "goût bourgeois ». Martine de Rougemont insiste sur l'abondance des théâtres non professionnels dans la France du XVIII ${ }^{\mathrm{e}}$ siècle, qui « accompagnent toute l'activité théâtrale, $[. .$.$] , la précèdent souvent, la doublent$ et la couronnent », et marquent tout autant un désir toujours plus fort de distinction sociale entre les amusements des grands, les cercles pornographiques parisiens, les sociétés bourgeoises ou associations populaires ${ }^{85}$ (jusqu'au théâtre rural, sur lequel Georges Hérelle a proposé une synthèse marquant sa valeur religieuse et communautaire, son rôle de conservatoire des idiomes et des cultures dans les pays de langue d'oc, en Savoie, dans le Dauphiné, en Bretagne ou en Flandre, où il donne lieu à des concours laïcs organisés autour d'une seule pièce classique réinterprétée par plusieurs troupes en langue vernaculaire ${ }^{86}$ ). Tous les genres se mêlent et s'abâtardissent dans ces jeux, quoique aristocratie et bourgeoisie affectionnent, le plus souvent dans l'intimité familiale, celle du pensionnat ou du salon, le théâtre d'éducation, qui a nourri les études récentes de MarieEmmanuelle Plagnol-Diéval ${ }^{87}$. Il est l'objet de conséquentes publications ou réimpressions des années 1750 à la Révolution : travaux de Mmes de Maintenon, de La Fite, de Genlis, du marquis de Saint-Marc, de Berquin, de Nougaret, de Gessner, de Garnier ou de Moissy. Réécritures rapides, démonstratives et explicatives, de thèmes connus mobilisant intrigues et personnages-types que l'on retrouve d'une pièce à l'autre, il oppose avec manichéisme et naïveté le bon et le méchant, la vertu et le vice, dans une suite éducative qui tient compte de l'âge des destinataires, des acteurs amateurs et valorise la civilité familiale. Il contribue à faire glisser la morale édifiante de la sphère religieuse vers l'espace laïc. Rompant avec une discrétion de bon ton, Mme de Genlis, jouera plusieurs mois, accompagnée de ses deux filles, dans le théâtre loué d'une société bourgeoise où se pressent jusqu'à cinq cents spectateurs, parfois payants : un narcissisme dont Mme de Staël appelera à se méfier dans La Sunamite ${ }^{88}$.

Cette expérience en général privée est prolongée par les sorties au théâtre. Le large spectre social des parterres des grands théâtres parisiens, où s'entasse un public si dense que les troubles sont inévitables et incontrôlables, toutes les règles de la civilité éventuellement bafouées, prouve notamment cette confluence et ses conséquences : "plutôt que de préserver le sens critique du détachement, [...] les spectateurs du parterre du XVIII ${ }^{\mathrm{e}}$ siècle insistaient sur la participation, de manière émotionnelle et physiquement explicite ", jusqu'à interrompre ou empêcher certaines représentations - d'où le souci d'ordre social que finissent par exprimer les projets pour les nouvelles salles de spectacles comme ceux de Wailly ou de Ledoux, offrant des places assises au public des premiers rangs et renvoyant ses éléments populaires au «poulailler » ${ }^{89}$. Irruption du public il y a bien, dans une rencontre sociale, déjà bien avancée avant et accélérée pendant la décennie révolutionnaire, entre le monde des boulevards et celui des scènes à privilèges. Issu du peuple et devenu maître, Jacques-Louis Ménétra, suffisamment introduit pour fréquenter les coulisses et ayant foulé lui-même la scène en amateur, emmène sa famille voir les Italiens ou le répertoire de l'Opéra-Comique, mais apprécie tout autant les danseurs de corde du boulevard du Temple ou les arlequinades de Chaumont ${ }^{90}$. Jusqu'aux éventails des maîtres de l'Académie de Saint-Luc ou des marchands d'estampes de la rue Saint-Jacques, tels que nous apprend à les ouvrir Georgina Letourmy, qui en rendent compte pour une clientèle désormais essentiellement

\footnotetext{
${ }^{84}$ Ibidem. Lettre $\mathrm{n}^{\circ} 48$ du 22 janvier 1789.

${ }^{85}$ Martine de Rougemont, La vie théâtrale en France au XVIIIe siècle, Paris, 1988, p. 297 et sqtes.

${ }^{86}$ Les Théâtres ruraux en France, Champion, 1930.

${ }^{87}$ Marie-Emmanuelle PlagnOL-DiEVAL, Madame de Genlis et le théâtre d'éducation au XVIIIe siècle, Oxford, 1997.

${ }^{88}$ M. de RougEMONT, op. cit., p. 306.

${ }^{89}$ Jeffrey S. RAVEL, «Le théâtre et ses publics : le parterre à Paris au XVIII ${ }^{\mathrm{e}}$ siècle », Revue d'histoire moderne et contemporaine, juillet - septembre 2002, p. 89-118; The Contested Parterre : Public Theater and French political Culture (1680 - 1791), New-York, 1999. Voir aussi Gregory BRown, A Field of Honor : Writers, Court Culture and Public Theater in French Literary Life from Racine to the Revolution, New-York, 2002.

${ }^{90}$ Daniel Roche, Jacques-Louis Ménétra, Journal de ma vie, op. cit., p. 317-318.
} 
bourgeoise. Ne représentent-ils pas fréquemment, à partir des années 1770, et comme le font du reste les boutons des vêtements masculins, des scènes du répertoire théâtral, notamment de l'opéracomique, sans omettre des références scénographiques ? Ne mêlent-ils pas aux promeneurs, aux courtisans, des personnages de la commedia dell'arte, Arlequin le premier? N'expriment-ils pas avec un souci pédagogique partisan, 1789 advenu, le verdict du public, mettant en exergue des pièces à succès comme Charles IX ou l'École des Rois, Nicodème dans la lune, les Deux petits savoyards, Brutus, Raoul, sire de Créqui, et accompagnant le dessin de commentaires qui explicitent la pièce ou la rattachent aux événements contemporains ${ }^{91}$ ? La critique majoritairement regrettera cette démocratisation du public et ce mélange des genres ; une fois celle-ci considérée comme achevée, elle y verra un avatar de la Révolution. Le fait est clair pour Fabien Pillet, fort de ses sympathies royalistes, qui fait imprimer en l'an IX La nouvelle lorgnette de spectacles (287 pages in-16 vendues un franc et cinquante centimes), dans laquelle il use de l'antiphrase assassine contre cent quatorze comédiennes et cent trente-sept comédiens de tout rang, particulièrement contre ceux des boulevards, pourtant volontairement brièvement évoqués. Il prétend au «bon goût », antithèse du «mauvais goût des spectateurs", du «gros public », des «manières provinciales » jurant avec les normes de la capitale - thème dont les auteurs eux-mêmes font classiquement leurs choux gras, pourfendant tous les Pourceaugnac de France et de Navarre. Il dit ses regrets pour le ton et les attitudes «trop bourgeois » ou trop boulevardiers de tel ou tel acteur (en lieu et place de la «noblesse » d'expression attendue), preuve d'un goût bourgeois construit bien davantage par ses pourfendeurs qu'en conscience par les principaux intéressés ${ }^{92}$. Car, avide de savoir et de comprendre, le monde divers des spectateurs est bien mieux formé que ne le prétendent ses contempteurs, prompts à défendre une gradation des civilités qui parcourt bel et bien jusqu'au monde des amateurs mais n'en exclut aucun.

Là encore, à bien lire les correspondances, l'observation vaut pour la province. Fils d'un avocat de Montluçon (Allier), Gilbert Favier prouvera son appétit tôt inculqué pour les arts de la scène partout où le porteront ses pas de volontaire dans les armées républicaines : il apprendra, pour mieux goûter les tréteaux européens, les langues des pays occupés, l'allemand et l'italien particulièrement, traduisant Les souffrances du jeune Werther ou assistant par exemple, en l'an VIII, à une représentation en langue vernaculaire de Misanthropie et repentir, de Kotzebue ${ }^{93}$. On sait Miette Tailhand sensible aux spectacles de la Comédie de Clermont-Ferrand, très fréquentée au demeurant et occasion d'un affichage social qui conduit l'art du paraitre ${ }^{94}$, voire à des rixes sanglantes entre loges et parterre, auxquelles Gaultier de Biauzat et Couthon avaient pu assister en 1780 et qui se reproduiront en l'an $\mathrm{V}^{95}$. Le même Biauzat n'avait pas oublié l'année précédente d'inciter ses concitoyens auvergnats, ses amis de la municipalité de Clermont-Ferrand surtout, à imiter la mise en scène de L'Offrande à la liberté, créée par Gossec et Méhul à l'Opéra de Paris, le 30 septembre $1792^{96}$.

Et que dire de l'acculturation assumée par ce simple volontaire aux frontières, fils de procureur et cousin de « Miette » Tailhand, obligé de rappeler sa sœur, actrice en herbe, à la raison,

\footnotetext{
${ }^{91}$ Georgina Letourmy, «L'éventail du succès : le théâtre mis en images à la veille de la Révolution », in Philippe Bourdin et Gérard LoubinOuX, La scène bâtarde, des Lumières au romantisme, Clermont-Ferrand, Presses universitaires Blaise Pascal, 2004, p. 221-240.

92 Philippe Bourdin, «La vulgarisation de la critique théâtrale au début du Consulat », in Annie CREPIN, Michel BIARD et Bernard GAINOT (dir.), La plume et le sabre. Mélanges Jean-Paul Bertaud, Paris, Publications de la Sorbonne, 2002, p. 147-161.

${ }_{93}$ Louis DuCHET, Deux volontaires de 1791. Les frères Favier de Montluçon. Journal et lettres, Montluçon, 1909. Héritier d'une sociabilité bourgeoise, Gilbert n'est pas moins sensible à Terpsichore et fondera à dessein une société de danse à Besançon, au sein de l'état-major où il est capitaine - le droit d'entrée en sera prohibitif.

${ }^{94}$ René BousCAYrol, Les lettres de Miette, op. cit. Lettre ${ }^{\circ} 25$ du 28 juin 1788.

95 André BossuAT, «Le théâtre à Clermont-Ferrand aux XVII ${ }^{\mathrm{e}}$ et XVIII ${ }^{\mathrm{e}}$ siècles », Revue d'histoire du théâtre, treizième année, tome II, 1961-1962; Philippe Bourdin, Des lieux, des mots, les révolutionnaires. Le Puy-de-Dôme entre 1789 et 1799, Clermont-Ferrand, Presses universitaires Blaise Pascal, 1995, p. 54.

${ }^{96}$ Archives départementales du Puy-de-Dôme, F 156 (78). Lettre de Biauzat aux administrateurs clermontois, 25 nivôse an IV (15 janvier 1796).
} 
lui imposant de reconnaître l'existence d'un plus grand et plus important théâtre, celui des opérations :

«Je ne m'étendrai pas davantage sur ce que nous pouvons souffrir. Le courage et l'espérance fait disparaître toutes nos souffrances. Tout en jouant ici mon rôle dans le tragique, naturel, je souhaite à tous nos parents et concitoyens la suite de leurs amusements civiques. J'espère qu'à mon retour, si je suis assez heureux pour le voir, je ne serai pas un acteur sans apprentissage. Nous n'avons point ici pour maître ni les Racine, ni les Corneille, ni les Voltaire ; mais, cependant, le théâtre ne laisse pas que d'être des plus fréquentés ${ }^{97}$.

La Révolution favorise, il est vrai, la création ou la récupération des comédies bourgeoises, dans le sillage bien souvent des clubs jacobins, autant qu'elle permet la multiplication des salles professionnelles ou semi-professionnelles ${ }^{98}$. Anne Hertert a mesuré l'impact de l'événement sur une ville telle que Besançon ${ }^{99}$. Les Bisontins sont peu familiarisés avec le théâtre lorsque arrive 1789 ; la salle de spectacles, pensée par Claude-Nicolas Ledoux et achevée en 1784, ne sert qu'occasionnellement. Une troupe professionnelle y est admise cette année-là, qui en 1791 entre en conflit avec la Société des Amis de la Constitution, s'émouvant de son refus de jouer des pièces patriotiques et obtenant finalement la fermeture de la salle à la fin de décembre 1792. Du club jacobin émerge alors une Société Dramatique, destinée à former l'opinion publique et à soulager l'indigence par les recettes espérées. Des amateurs la composent, qui obtiennent de la municipalité une salle et du bois de chauffage, et produisent leur premier spectacle le 20 janvier 1793 (Beverley, de Saurin). Quarante comédiens, dix musiciens se retrouvent pour jouer pendant trois ans une pièce chaque dimanche puis chaque décadi. Très strict, le règlement du 30 juin 1794 prévoit différentes conditions d'admission: des qualités morales appréciées par le comité de la section du candidat; des aptitudes au théâtre vérifiées par le comité d'examen de la Société dramatique ; une élection par l'assemblée générale des amateurs et l'approbation des officiers municipaux. Les épurations administratives et politiques entraînent cependant une instabilité de la troupe, d'autre part handicapée par la surreprésentation des hommes (83\% des artistes en moyenne, et parmi eux $11 \%$ d'adolescents), conséquence fâcheuse d'une mésentente avec la Société des Amies de la Liberté et de l'Égalité fondée en 1792. Autant qu'il soit mesurable (c'est-à-dire pour la moitié de l'effectif), le recrutement privilégie les tenants de l'autorité révolutionnaire (administrateurs et leurs enfants, commissaires des sections, hommes de loi, négociants, enseignants), que ce soit en 1794 ou en 1796. Le répertoire, varié, épouse les mutations des gouvernements : avant la Terreur, les textes corrigés par la Comédie française servent de référence et l'on produit Voltaire, Molière, Rousseau, Regnard, Grétry, Monsigny, Monvel et Dalayrac; pendant la Terreur dominent les pièces patriotiques (Bara, Le Départ des volontaires, La Parfaite Égalité) et anti-religieuses (Les Visitandines de Picard et Devienne, par exemple) ; après le 9 Thermidor sont données des œuvres anti-jacobines, comme L'Intérieur des Comités révolutionnaires de Ducancel ou Les Suspects et les fédéralistes de Martainville. Le refus de jouer des airs patriotiques en 1796 rend la troupe suspecte aux yeux des autorités directoriales; sauvée par le regain royaliste de 1797, elle doit subir dès l'année suivante la concurrence d'une troupe républicaine. Peut-on mieux marquer que par cet exemple le passage permanent pour plusieurs des citoyens impliqués du dilettantisme culturel au dilettantisme politique?

La Comédie de Riom, fondée dans la dernière décennie de l'Ancien Régime, s'avère pareillement active ${ }^{100}$. Ses membres, autant que permette de le dire le faible échantillon renseigné (treize personnes), ont trente ans en moyenne, les femmes étant plus jeunes que les hommes de trois

\footnotetext{
${ }^{97}$ René BousCAYrol, «Deux lettres d'un volontaire riomois à sa mère », Revue d'Auvergne, 1985, n 2, p. $249-254$. Lettre de Gilbert Chabory du 3 nivôse an III (23 décembre 1794).

${ }^{98}$ Philippe BoURDIN, «Le théâtre, les amateurs, la Révolution », in La scène bâtarde entre Lumières et Romantisme, op. cit., p. 241-254.

99 Anne HERTERT, «La Société dramatique de Besançon et le personnel révolutionnaire bisontin de 1793 à 1796 », in Théâtre et Révolution, Actes du colloque international de Besançon (juin 1988), $\mathrm{n}^{\circ}$ spécial des Annales littéraires de l'Université de Besançon, Paris, s.d., p. 35-43

${ }^{100}$ Philippe Bourdin, «Les théâtres de société sont-ils solubles dans la Révolution ? L'exemple de l'Auvergne », in Marie-Emmanuelle Plagnol-Dieval et Dominique Quero (dir.), Les théâtres de société au XVIIIe siècle, Études sur le XVIII ${ }^{e}$ siècle, Éditions de l'Université de Bruxelles, 2005, p. 157-168.
} 
années. Ceux-ci sont issus des mondes de la robe (juges, procureurs, avocats, greffier même représentent $54 \%$ au moins des acteurs) et du négoce riomois (au moins $21 \%$ ), «bourgeois de la ville », «parents ou amis » selon leur propre définition ${ }^{101}$, tenus par des liens professionnels, familiaux (mariages, cousinages, fraternités) ou de voisinage. Étonnons-nous de cette pratique théâtrale chez des jeunes gens dont l'éducation a été confiée aux brillants Oratoriens du cru, qui réprouvaient de tels jeux, et parions sur le besoin de distinction sociale qui n'épargne pas plus les milieux jansénistes que partie de la noblesse de cour, ou, au-delà du mimétisme comportemental, sur l'intérêt suscité par un art qui fédère partout des publics de plus en plus divers. Les robins tiennent à Riom le haut du pavé et monopolisent les deux tiers des fonctions municipales. C'est donc naturellement que les deux tiers des comédiens amateurs occupent sous la Révolution, parfois de manière continue, d'importantes fonctions électives locales (municipalités de la commune, du district, tribunaux en l'an III, mais certains ont servi le gouvernement révolutionnaire sous les représentants Couthon et Monestier). Ils comptent aussi parmi les fondateurs du club jacobin et au moins la moitié participent à la société populaire de l'an II. Se réunissant en novembre 1794 dans la «Société des Amis du Théâtre » pour donner leurs premières représentations publiques ${ }^{102}$, ils se veulent, selon les valeurs mises à la mode dans les années 1780 , coterie philanthropique n'accueillent-ils pas, du reste, l'épouse du régisseur du dépôt de mendicité ? -, et destinent leurs recettes aux indigents. Cette transformation correspond parfaitement à un modèle ailleurs éprouvé (Dijon, Troyes ${ }^{103}$ ) et aux encouragements accordés par le gouvernement révolutionnaire pour que se développent les scènes patriotiques, placées au cœur du dispositif de propagande républicaine comme « écoles primaires pour adultes ».

Le succès de la Société des Amis du Théâtre crée un effet de mode. Est-ce le risque de concurrence qui pousse la Société à se doter d'un règlement pour s'installer solidement dans les murs ? Adopté par la municipalité le 7 pluviôse an III (26 janvier 1795), après avoir été élaboré avec les amateurs le mois précédent, celui-ci reprend en bien des points (et en trente-trois articles...) les exigences imposées aux professionnels. Il planifie les prérogatives de la direction, les rôles et les entrées des artistes, la gestion des répétitions et des défaillances, jusqu'aux amendes infligées pour travail insuffisant. La collégialité du fonctionnement est cependant affirmée. On entre dans la troupe par cooptation de cinq membres - cooptation qui renvoie aux modèles académiques et maçonniques. L'ordre interne est autogéré. On choisit le répertoire en assemblée générale selon de lourdes procédures. Les missions patriotiques, la participation aux fêtes civiques surtout, le sérieux tout autant et, rompant avec la réalité contemporaine, on tente d'instaurer une distance symbolique entre scène et parterre, entre l'imaginaire et le réel. Sans doute le souci d'ordre moral n'est-il pas absent de l'interdiction faite aux enfants de fréquenter le théâtre s'ils ne sont accompagnés, aux curieux d'assister aux répétitions; il faut notamment répondre aux préventions des mères de famille contre les dangers de la mixité entre cour et jardin, en un temps où l'actrice doit combattre la réputation d'hétaïres de luxe des comédiennes les plus renommées. Bref, on reste dans le domaine de la mondanité, d'une sociabilité qui s'interrompt une fois l'été venu et les notables partis pour leurs résidences secondaires. Ces usages qui s'exportent d'une ville à l'autre, en fonction des alliances économiques et familiales. Miette Tailhand, l'une des héroïnes des jeux riomois, a donc assisté en 1793 à Issoire, sous la houlette de Jean-Baptiste Reymond, « directeur de la comédie bourgeoise » de cette ville commerçante qui compte moins de 5000 habitants, à un Brutus (de Voltaire ou de Dorat ?) qu'il mettait en scène dans une petite salle où la jeune Riomoise, considérée comme affiliée, selon des termes empruntés au monde académique ou maçonnique, avait été installée à la place d'honneur. Si la jeune fille lui adresse ses éloges, il n'en va pas sans une certaine condescendance chez une demoiselle moins sûre de son art que de la supériorité

\footnotetext{
${ }^{101}$ René BousCAYrol, Les lettres de Miette Tailhand-Romme..., op. cit. Lettres 96 et 98, septembre et novembre 1794.

102 René BousCAYROL, Les lettres de Miette Tailhand-Romme..., op. cit. Lettre 97 du troisième trimestre 1794.

103 Clothilde Trehorel, Le théâtre de Dijon de 1789 à 1810, maîtrise de l'Université de Bourgogne, dirigée par Christine LAMARRE, octobre 1999, p. 98 ; Jeff HORN, « La lutte des factions au théâtre de Troyes sous le Directoire », in Philippe BOURDIN et Bernard GAINOT, La République directoriale, actes du colloque international de Clermont-Ferrand (22, 23, 24 mai 1997), Clermont-Ferrand - Paris, 1998, tome II, p. 679-690.
} 
intellectuelle et professionnelle de ses parents et amis, consciente aussi d'une gradation dans les exercices de civilité et de la valeur des amateurs riomois. Miette ne refuse-t-elle pas de déchoir en partageant ses talents avec ceux des Issoiriens ? Reymond voulant absolument lui faire accepter un rôle, elle repoussa ainsi « un honneur qui aurait compromis la dignité de notre société et peut-être [sa] réputation ${ }^{104}$...

\section{Conclusion}

Si les bourgeoisies trouvent peu ou prou, avec la décennie révolutionnaire, des responsabilités politiques à la mesure de leurs talents, la Révolution augmente en proportion leur souci de représentation et de distinction à l'œuvre durant le siècle. La revendication du goût, et si possible du bon, est de ce point de vue un marqueur fort des hiérarchies qui s'ordonnent au fil des événements. Au souci d'égalité promu par une minorité active en l'an II, qui se traduit symboliquement par l'unité nationale recherchée dans le vêtement - fût-ce au prix d'une identification forte de celui des magistrats, comme le voulaient les utopies sociales -, par le pain ou la gamelle communs, s'opposent les portraits des puissants d'un jour, l'affichage des parvenus, rompant avec une culture de la discrétion et de la modération qui reste fortement présente en province. La séparation d'avec la «populace », distinguée du peuple, affirmée souvent dans les récits d'histoire immédiate, n'est pas évidente en tout lieu et toutes circonstances (le théâtre, par exemple), mais une abondante littérature (traités de cuisine, critiques des spectacles, analyses des œuvres peintes) la revendique et s'emploie pourtant à instaurer des mondes incompatibles. Cette incompatibilité est parfois entrée dans les faits : c'est Miette Tailhand interdite de carnaval par sa grand-mère dans le même temps où Mercier constate le mépris de la bourgeoisie parisienne pour ce genre d'amusement ${ }^{105}$. Mais la mondanité bourgeoise sécrète ses propres hiérarchies, sa conscience de castes : quoi de commun entre les «nouveaux riches » et la famille Romme ? Quoi de commun, semble encore une fois dire Miette, entre les goûts et les pratiques de bourgeoisies provinciales issues de villes de taille et de sociologie différentes ? Seuls semblent finalement plaider pour un «goût bourgeois » ceux qui le vitupèrent et le réduisent au mélange des genres, à la grossière influence de la rue, et partant aux mœurs et à l'art révolutionnaires en général, les Pillet, les Grimod de la Reynière. Au crépuscule du XVIII ${ }^{\mathrm{e}}$ siècle, dans un moment de recomposition politique et culturelle, ils en nourrissent leurs imprécations au service des anti-Lumières, et inaugurent un thème dont se saisiront d'autant plus volontiers les romanciers que la liste des «notables » impériaux paraît à son tour, trompeusement, instaurer une élite unifiée.

Philippe BOURDIN

Centre d'histoire «Espaces et Cultures » Université Clermont-Auvergne

\footnotetext{
${ }^{104}$ René BousCAYrol, Les lettres de Miette Tailhand-Romme..., op. cit. Lettre $\mathrm{n}^{\circ}$ 83, septembre 1793.

${ }^{105}$ Ibidem, Lettre $\mathrm{n}^{\circ} \mathrm{du}$ « J'entends de tous côtés une musique bruyante qui accompagne les masques qui courent les rues; les cris de joie que les enfans jettent en voyant les caricatures excitent ma curiosité. Je voudrais qu'il me fut permis de me mettre à la fenêtre et d'en rire comme les autres. Ma grand'mère me le défend et je me régale d'un beau sermon. Plus attentive au son des instruments qu'aux leçons de morale qu'elle me donne, je répète tout bas les airs qu'on joue dans la rue ».
} 